Surveys in Differential Geometry XIV

\title{
Riemann surfaces, integrable hierarchies, and singularity theory
}

\author{
Yongbin Ruan
}

\section{Introduction}

In 1991, a celebrated conjecture of Witten [Wi1] asserted that the intersection theory of Deligne-Mumford moduli space is governed by KdVhierarchies. His conjecture was soon proved by Kontsevich $[\mathbf{K o}]$. Since then, the Witten-Kontsevich theorem has introduced the seemingly alien concept of integrable hierarchies to the geometry. Immediately after, a great deal of effort was spent in investigating other integrable hierarchies in GromovWitten theory. A much studied example is 2-Toda hierarchies for $\mathbb{P}^{1}$ by Okounkov-Pandharipande. It was generalized recently to orbifold $\mathbb{P}^{1}[\mathbf{J}]$, $[\mathbf{M T}],[\mathbf{P R}]$. A famous problem of similar flavor is the Virasoro constraint for Gromov-Witten theory for an arbitrary target. The common characteristics of these problems are: (1) all of them are very difficult; (2) all of them are mysterious. In particular, the choice of integrable hierarchies seems to be matter of luck and there is no general pattern to predict the hierarchies for a given geometrical problem. Therefore, it is particularly important to explore the relation of integrable hierarchies to Gromov-Witten theory systematically. This is the main focus of this survey.

In fact, this question was very much in Witten's mind when he proposed his famous conjecture in the first place. Around the same time, he also proposed a sweeping generalization of his conjecture [Wi2, Wi3]. The core of his generalization is a remarkable first order nonlinear elliptic PDE associated to an arbitrary quasihomogeneous singularity. During the last few years, Witten's generalization has been explored and a new Gromov-Witten type theory has been constructed by Fan-Jarvis-Ruan [FJR1, FJR2, FJR3]. In particular, Witten's conjecture for ADE-integrable hierarchies has been verified. It is important to mention that the geometry behind these integrable

Partially supported by a NSF grant.

(C)2009 International Press 
hierarchies concerns the special cycles of $\overline{\mathcal{M}}_{g, k}$ and fits well into the main theme of this volume.

This is a survey article aiming at two purposes. The detailed construction of Fan-Jarvis-Ruan-Witten is a long one. It will take an interested reader a while to penetrate all the technical details. In this article, we will omit all the technical details and focus on the motivation and ideas. More importantly, ADE-singularities comprise a very special class of singularities. There is a vast territory beyond ADE-singularities where classical integrable hierarchies will play an important role and perhaps new integrable hierarchies should be constructed. Furthermore, mirror symmetry has made a surprising appearance in integrable hierarchies problems. I feel that this is a good opportunity for young people to expand their horizons and I hope that this article will be helpful. In particular, we will discuss some future directions in the last section of the paper.

This "quantum" singularity theory of Fan-Jarvis-Ruan-Witten has farreaching applications to other problems such as the Landau-Ginzburg/ Calabi-Yau correspondence [Wi4, CR1]. It is under rapid development. We hope to come back to it at another occasion.

The paper is organized as follows. In section two, we will sketch the construction of integrable hierarchies from integrable representations of infinite dimensional Lie algebras. In section three, we will explain Witten's original conjecture for $\mathrm{KdV}$. The main part is section four where we will introduce the Fan-Jarvis-Ruan-Witten's theory for quasihomogenenous singularities. In section five, we sketch the proof of Witten's ADE-hierarchies conjecture. In particular, a modification of Witten's conjecture for $D_{n}$ ( $n$ odd) is needed due to mirror symmetry. In section six, we will describe a further generalization of Witten's conjecture for singularities beyond ADE.

\section{Integrable hierarchies and representation of Kac-Moody algebras}

Integrable hierarchies is a diverse subject and there are many different points of views. Roughly speaking, it is a system of differential equations for a function of infinitely many time variables $F\left(x, t_{1}, t_{2}, \ldots\right)$ where $x$ is a spatial variable and $t_{1}, t_{2}, \ldots$, are time variables. The PDE is a system of evolution equations of the form

$$
\frac{\partial F}{\partial t_{n}}=R_{n}\left(x, F_{x}, F_{x x}, \ldots\right),
$$

where $R_{n}$ is a polynomial. Usually, $R_{n}$ is constructed recursively.

The traditional approach of integrable hierarchies concerns its bi-Hamiltonian structure, Lax pair and so on. Here, we briefly sketch it from the point of view of the Hirota bilinear equation of integrable representations

of infinite dimensional Lie algebras. This point of view naturally leads to ADE-hierarchies. 
2.1. Fermonic Fock space and KP-hierarchy. The most famous example of this point of view is the interpretation of the KP-hierarchy in terms of the Hirota equation of the fermonic (infinite wedge) representation of $G L_{\infty}$ by the Kyoto school [DJKM1, DJKM2], [DJKM3, DJKM4].

To motivate the construction, we start from a geometric problem of the Plücker embedding of the Grassmannian $G(n, k)$. Let $W \subset \mathbb{C}^{n}$ be a $k$ dimensional subspace of $\mathbb{C}^{n}$. Its Plücker coordinate is $v_{1} \wedge v_{2} \wedge \cdots \wedge v_{k} \in \wedge^{k} \mathbb{C}^{n}$ for a basis $v_{1}, \ldots, v_{k}$ of $W$. If $v_{1}^{\prime}, \ldots, v_{k}^{\prime}$ is another basis, $v_{1}^{\prime} \wedge \cdots \wedge v_{k}^{\prime}=$ $c v_{1} \wedge \cdots \wedge v_{k}$. Therefore, it uniquely determines a point in $\mathbb{P}\left(\wedge^{k} \mathbb{C}^{n}\right)$ which defines an embedding of $G(n, k)$ into $\mathbb{P}\left(\bigwedge^{k} \mathbb{C}^{n}\right)$. Now, we want to determine the image of the embedding. There are two ways to do it. The first method is to use the action of $G L(n, \mathbb{C})$ on $\mathbb{C}^{n}$. It induces an action on $G(n, k)$ as well as an action on $\bigwedge^{k} \mathbb{C}^{n}$. The image of the Plücker embedding is the set of points of $\wedge^{k} \mathbb{C}^{n}$ of the form $v_{1} \wedge \cdots \wedge v_{k}$. Since $G L(n, \mathbb{C})$ acts transitively on $G(n, k)$, all of them can be generated by the $G L(n, \mathbb{C})$-action on $\bigwedge^{k} \mathbb{C}^{n}$ from a single element, say $e_{1} \wedge \cdots \wedge e_{k}$ where $e_{1}, \ldots, e_{n}$ is the standard basis of $\mathbb{C}^{n}$. Now, we write down the action of $G L(n, \mathbb{C})$ explicitly. Any matrix can be written as a linear combination of $E_{i j}$ with a single entry at $(i, j)$. It is easy to check that $E_{i j}$ acts on $\bigwedge^{k} \mathbb{C}^{n}$ by

$$
r\left(E_{i j}\right)=\psi_{i} \psi_{j}^{*}
$$

where

$$
\psi_{i}\left(v_{1} \wedge \cdots \wedge v_{k}\right)=e_{i} \wedge v_{1} \wedge \cdots \wedge v_{k}, \psi_{j}^{*}\left(v_{1} \wedge \cdots \wedge v_{k}\right)=i_{e_{j}}\left(v_{1} \wedge \cdots \wedge v_{k}\right),
$$

where $i_{e_{j}}$ is the contraction by $e_{j}$.

The second method is to write down a set of equations whose zero set is the image. It is also well known in algebraic geometry that the image is given by a set of quadratic equations (Plücker equations)

$$
\sum_{i} \psi_{i}(\tau) \otimes \psi_{i}^{*}(\tau)=0
$$

Now, we generalize the above setting to the infinite dimensional situation. We replace $\mathbb{C}^{n}$ by an infinite dimensional vector space $V$ generated by a basis $e_{j}$ for $j \in \mathbb{Z} . \bigwedge^{k} \mathbb{C}^{n}$ is replaced by the following infinite wedge space $\bigwedge^{\infty} V$, linear combinations of vectors of the form

$$
e_{i_{0}} \wedge e_{i_{1}} \wedge \cdots
$$

such that $i_{n}=-n$ for $n \ll 0$. The above object is called a semi-infinite monomial.

We start from constructing the action of Lie algebra $\tilde{g l}_{\infty}$. One has to be more careful since two arbitrary $\mathbb{Z} \times \mathbb{Z}$ matrices may not be able to be multiplied. A $j$-th diagonal matrix means $\sum_{i \in \mathbb{Z}} a_{i} E_{i, i+j}$. We require an element of $\tilde{g l} l_{\infty}$ to be a sum of finitely many diagonal matrices. It is easy to 
see that the product of two such matrices is of the same type and hence we can define its Lie bracket. $\tilde{g} l_{\infty}$ acts on $V$ and induces an action on $\bigwedge^{\infty} V$.

$\wedge^{\infty} V$ is also a so-called highest weight representation of the central extension $\hat{g l}_{\infty}$ of $\tilde{g} l_{\infty}$. To see this, it is enough to consider the action $r\left(E_{i j}\right)$. $r\left(E_{i j}\right)$ for $i \neq j$ works well in the infinite dimensional case. But we have to modify the action of $E_{i i}$ as

$$
r\left(E_{i i}\right)=: \psi_{i} \psi_{i}^{*}:= \begin{cases}\psi_{i} \psi_{i}^{*} & \text { for } i \leq 0 \\ \psi_{i}^{*} \psi_{i} & \text { for } i>0\end{cases}
$$

We also use $: \psi_{i} \psi_{j}^{*}$ : to denote $\psi_{i} \psi_{j}^{*}$ even for $i \neq j$. With the above modification, we no longer get a representation of $\tilde{g l}{ }_{\infty}$. Instead, we obtain a representation of certain central extension $\hat{g l} l_{\infty}$. Finally, we replace $e_{1} \wedge e_{2} \cdots \wedge e_{k}$ by

$$
|0\rangle=e_{0} \wedge e_{-1} \wedge \cdots \text {. }
$$

We call $|0\rangle$ the vacuum vector. Furthermore, every other vector can be generated from $|0\rangle$ by repeatedly applying $r\left(E_{i j}\right)$ for $i>j$. For this reason, $\bigwedge^{\infty} V$ is also called a highest weight representation and $|0\rangle$ is called a highest weight vector. $\wedge^{\infty} V$ is often referred to as the fermionic representation of $g l_{\infty}$ or fermionic Fock space. Finally, we define $\widetilde{G L}_{\infty}$ to be the Lie group of $\tilde{g l} l_{\infty}$. We remark that for $R \in \widetilde{G L}_{\infty}, r(R)(|0\rangle)$ is an infinite sum of semi-infinite monomials. Namely, we really should think that $\widetilde{G L}_{\infty}$ acts on the completion of $\bigwedge^{\infty} V$.

Now, we are interested in the infinite Grassmanian as the orbit of $\Omega=$ $\widetilde{G L}_{\infty}(|0\rangle)$. The analogue of the Plücker equation is

$$
\sum_{j \in \mathbb{Z}} \psi_{j}(\tau) \otimes \psi_{j}^{*}(\tau)=0
$$

The key to make contact with integrable hierarchies is the boson-fermion correspondence $\bigwedge^{\infty} V \cong \mathbb{C}\left[x_{1}, x_{2}, \ldots,\right]$. The easiest way to understand this correspondence is to use the following Heisenberg subalgebra of $\widetilde{g l}_{\infty}$. Let $\wedge_{k}=\sum_{i} E_{i, i+k}$. One can show that $\bigwedge^{\infty} V$ is generated by applying $r\left(\wedge_{k}\right)$ for $k<0$. Now, we define a representation of $\wedge_{k}$ on $\mathbb{C}\left[x_{1}, x_{2}, \ldots,\right]$ by,

$$
\wedge_{k}=\frac{\partial}{\partial x_{k}} ; \wedge_{-k}=k x_{k} \cdot k>0
$$

The boson-fermion correspondence is given by mapping $|0\rangle$ to 1 and matching the representation of $\wedge_{k}$.

With this identification, an element $\tau \in \Omega$ is identified with a power series (also denoted by $\tau$ ) in $\mathbb{C}\left[x_{1}, x_{2}, \ldots\right]$. The Plücker equation is identified with a series of differential equations called the Hirota equation. The relation between the Hirota equation and the KP-hierarchies is that $\tau=e^{F}$ where $F$ is the solution of the KP-hierarchies. For our purpose, we do not need to know the explicit form of the KP-hierarchies. It is equally sufficient for us to work with the corresponding Hirota equation. 
There is a more compact expression of the Hirota equation using the vertex operator formulation. Then, we need to make sense of the fermionic operators $\psi_{i}, \psi_{j}^{*}$ individually. We define $\bigwedge_{m}^{\infty} V$ for $m \in \mathbb{Z}$ as the vector space generated by the semi-infinite monomials

$$
e_{i_{0}} \wedge e_{i_{1}} \wedge \cdots
$$

with $i_{k}=k+m$ for $k \ll 0$. $\bigwedge_{m}^{\infty} V$ has its own vacuum vector

$$
e_{m} \wedge e_{m-1} \wedge \cdots \text {. }
$$

It is clear that $\bigwedge^{\infty} V=\bigwedge_{0}^{\infty} V$. Then, let $\mathcal{V}=\bigoplus_{m}\left(\bigwedge_{m}^{\infty} V\right)$, and then

$$
\psi_{i}, \psi_{j}^{*}: \mathcal{V} \rightarrow \mathcal{V}
$$

The boson-fermion correspondence defines an isomorphism

$$
\mathcal{V} \cong \mathbb{C}\left[x_{1}, x_{2}, \ldots, z, z^{-1}\right]=\bigoplus_{m} z^{m} \mathbb{C}\left[x_{1}, x_{2}, \ldots\right] .
$$

Let us introduce the generating series of operators

$$
X(u)=\sum_{j \in \mathbb{Z}} u^{j} \psi_{j}, X^{*}(u)=\sum_{j \in \mathbb{Z}} u^{-j} \psi_{j}^{*}
$$

Under the boson-fermion correspondence, $X(u) \rightarrow \Gamma(u), X^{*}(u) \rightarrow \Gamma^{*}(u)$, where

$$
\begin{aligned}
\left.\Gamma(u)\right|_{z^{m} \mathbb{C}\left[x_{1}, x_{2}, \ldots\right]} & =u^{m+1} z \exp \left(\sum_{j \geq 1} u^{j} x_{j}\right) \exp \left(-\sum_{j \geq 1} \frac{u^{-j}}{j} \frac{\partial}{\partial x_{j}}\right), \\
\left.\Gamma^{*}(u)\right|_{z^{m} \mathbb{C}\left[x_{1}, x_{2}, \ldots\right]} & =u^{-m} z^{-1} \exp \left(-\sum_{j \geq 1} u^{j} x_{j}\right) \exp \left(\sum_{j \geq 1} \frac{u^{-j}}{j} \frac{\partial}{\partial x_{j}}\right) .
\end{aligned}
$$

This leads to a formulation of the Hirota equation in $\mathbb{C}\left[x_{1}, x_{2}, \ldots\right]$ as the vanishing of the $u^{0}$-term in the expression

$$
u \exp \left(-\sum_{j \geq 1} 2 u^{j} y_{j}\right) \exp \left(\sum_{j \geq 1} \frac{u^{-j}}{j} \frac{\partial}{\partial y_{j}}\right) \tau(x-y) \tau(x+y) .
$$

2.2. Affine Kac-Moody algebras and their integrable hierarchies. The above picture has been generalized extensively for many different types of infinite dimensional Lie algebra/integrable hierarchies. The most relevant one for us is the one constructed by Drinfeld-Sokolov [DS] and KacWakimoto $[\mathbf{K W}]$. Both of them are constructed out of the integrable representations of affine Kac-Moody algebras. The Drinfeld-Sokolov version is formulated in terms of the traditional integrable system approach. The KacWakimoto version is formulated along the lines of the Hirota equation. Fortunately, they are equivalent by the work of Hollowood and Miramontes [HM]. 
Here, we describe Kac-Wakimoto's approach. It is clear that the previous construction depends on three pieces of data.

(i) An infinite dimensional Lie algebra $g l_{\infty}$.

(ii) An integrable highest weight representation $\bigwedge^{\infty} V$ of $\tilde{g} l_{\infty}$.

(iii) The boson-fermion correspondence $\bigwedge^{\infty} V \cong \mathbb{C}\left[x_{1}, x_{2}, \ldots\right]$.

In Drinfeld-Sokolov-Kac-Wakimoto's generalization, they replace $g l_{\infty}, \wedge^{\infty} V$ by an affine Kac-Moody algebra and its integrable highest weight representation $V$. Unfortunately, there is no boson-fermion correspondence in general. Thus, they replace it by a vertex operator construction $R$ of $V$. In these cases, they have written down the corresponding Hirota equation for the orbit $G|0\rangle$ of the corresponding group $G$.

The affine Kac-Moody algebras are classified by affine Dynkin diagrams. They come in two types, the untwisted ones and twisted ones. The untwisted ones are labelled by $A_{n}^{(1)}(n \geq 1), B_{n}^{(1)}(n \geq 3), C_{n}^{(1)}(n \geq 2), D_{n}^{(1)}(n \geq 4), G_{2}^{(1)}$, $F_{4}^{(1)}, E_{6}^{(1)}, E_{7}^{(1)}, E_{8}^{(1)}$. The affine Kac-Moody algebra is the central extension of the loop algebra of the corresponding simple Lie algebra. Others are labelled by $A_{n}^{(2)}(n \geq 2), D_{n+1}^{(2)}(n \geq 2), E_{6}^{(2)}, D_{4}^{(3)}$. The second types are certain orbifoldings of untwisted ones. The $(V, R)$ also have many choices. Thus, we obtain many different types of integrable hierarchies. For example, for ADE-affine untwisted Kac-Moody algebras, Kac-Wakimoto constructed two types of hierarchies, the principal picture and the homogeneous picture from the different realizations of the basic representation. When we talk about the principal picture of the untwisted series, we will simply refer to it as the hierarchies of the corresponding type. Furthermore, if a power series $F$ satisfies the Hirota equation, we will say that it is a $\tau$-function of the corresponding hierarchy.

The explicit formula is not important for our purposes. It is an extremely interesting problem whether all these classical integrable hierarchies can be realized in geometry such as the theory of Fan-Jarvis-Ruan-Witten for quasihomogeneous singularities.

It is clear that we do not have to restrict ourselves to affine Kac-Moody algebras. The same general idea should work in much greater generality. By going beyond affine cases, we have already entered the frontier of the subject of the representation theory of infinite dimensional Lie algebras. The affine case is in some sense corresponding to $\mathbb{C P}^{1}$. A very interesting case is the elliptic hierarchies [DJKM5] associated to elliptic curves. An interesting question is if they are the hierarchies that govern the theory of Fan-JarvisRuan-Witten for the elliptic singularities $P_{8}, X_{9}, J_{10}$ (see the last section for more discussion).

\section{Integrable hierarchies in geometry}

The first appearance of integrable hierarchies in Gromov-Witten theory is the $\mathrm{KdV}$-hierarchy. Its geometric counterpart is the intersection theory on 
the moduli space of stable Riemann surfaces $\overline{\mathcal{M}}_{g, k}$. The latter can be treated as the Gromov-Witten theory of the zero dimensional manifold. Let's review it in more detail.

Here, $\overline{\mathcal{M}}_{g, k}$ is the moduli space of isomorphism classes of genus $g$, stable, nodal Riemann surfaces with $k$-ordered marking. $\overline{\mathcal{M}}_{g, k}$ is a central object in algebraic geometry and has been studied intensively for decades. It is a smooth complex orbifold of dimension $3 g-3+k$. It is important to mention that $\overline{\mathcal{M}}_{g, k}$ is only well-defined in the so-called stable range $2 g+k \geq 3$. Over $\overline{\mathcal{M}}_{g, k}$, each marked point $x_{i}$ naturally defines an orbifold line bundle $L_{i}$ whose fiber at $C$ is $T_{x_{i}}^{*} C$. Let $\psi_{i}=C_{1}\left(L_{i}\right)$. One can define intersection number for $\psi_{i}$ classes.

$$
\left\langle\tau_{l_{1}}, \tau_{l_{2}}, \ldots, \tau_{l_{k}}\right\rangle_{g}=\int_{\overline{\mathcal{M}}_{g, k}} \prod_{i} \psi_{i}^{l_{i}} .
$$

Here, we define it to be zero unless $\sum_{i} l_{i}=3 g-3+k$. Now, we assemble it into the generating function

$$
\mathcal{F}^{g}\left(t_{0}, t_{1}, \ldots\right)=\sum_{k \geq 0} \frac{t_{l_{1}} \cdots t_{l_{k}}}{k !}\left\langle\tau_{l_{1}}, \tau_{l_{2}}, \ldots, \tau_{l_{k}}\right\rangle .
$$

$\mathcal{F}$ is a formal power series in infinitely many variables $t_{0}, t_{1}, \ldots$, . Then, we introduce the total descendant potential function

$$
\mathcal{D}=\exp \left(\sum_{g \geq 0} h^{g-1} \mathcal{F}^{g}\right) .
$$

$\mathcal{D}$ admits a geometric interpretation as the generating function of intersection numbers for disconnected stable Riemann surfaces.

We perform the dilaton shift

$$
q_{i}= \begin{cases}t_{i}, & i \neq 1 \\ t_{1}-1, & \text { otherwise }\end{cases}
$$

By the so-called dilaton equation, $\mathcal{F}^{g}$ is a homogeneous power series of degree $2-2 g$ in the new variables $q_{i}$. A central problem in mathematics and physics is to compute $\mathcal{F}^{g}$ or $\mathcal{D}$. We can try to write them as the combination of known functions such as exponential, trigonometric or more generally infinite products such as modular forms or hypergeometric functions. If this happens, we say that $\mathcal{F}^{g}$ or $\mathcal{D}$ has a closed formula. Unfortunately, this almost never happens for Gromov-Witten theory. The next best situation is to find the differential equations which it satisfies. We hope to find enough equations from which $\mathcal{F}^{g}$ or $\mathcal{D}$ will be uniquely determined. Ideally, these equations are determined by the classical geometry of the problem. It would be more striking if they come from entirely different sources. The celebrated Witten-Kontsevich theorem is one of such examples.

TheOREM 3.0.1. $\mathcal{D}$ is a $\tau$-function of the KdV-hierarchy. 
REMARK 3.0.2. $\mathcal{D}$ is uniquely determined by the $\mathrm{KdV}$-hierarchy together with the so-called string equation.

Since $\mathrm{KdV}$ is just the first example of a family of hierarchies, it is natural to ask about the underlying geometry of other integrable hierarchies. The basic idea is to consider more general intersection numbers $\int_{D} \prod_{i} \psi_{i}^{l_{1}}$ for a cycle $D \in H_{*}\left(\overline{\mathcal{M}}_{g, k}, \mathbb{Q}\right)$. This leads to the interesting subject of special cycles of $\overline{\mathcal{M}}_{g, k}$. Furthermore, we require $D$ to satisfy some general properties which are captured by the notion of cohomological field theory $[\mathbf{K M}]$.

Recall that there are several canonical morphisms between the $\overline{\mathcal{M}}_{g, k}$.

\section{Forgetful Morphism:}

$$
\pi: \overline{\mathcal{M}}_{g, k+1} \rightarrow \overline{\mathcal{M}}_{g, k}
$$

by forgetting the last marked point $x_{k+1}$. Here, we assume that $2 g+k \geq 3$. Furthermore, $\pi$ is the universal curve.

\section{Gluing the tree:}

$$
\rho_{\text {tree }}: \overline{\mathcal{M}}_{g_{1}, k_{1}+1} \times \overline{\mathcal{M}}_{g_{2}, k_{2}+1} \rightarrow \overline{\mathcal{M}}_{g_{1}+g_{2}, k_{1}+k_{2}} .
$$

\section{Gluing the loop:}

$$
\rho_{\text {loop }}: \overline{\mathcal{M}}_{g, k+2} \rightarrow \overline{\mathcal{M}}_{g+1, k}
$$

Suppose that $H$ is a graded vector space with a nondegenerate pairing $\langle$. and a degree zero unit 1 . To simplify the signs, we assume that $H$ has only even degree and the pairing is symmetric. When $H$ has odd degree elements, everything become "super" and we leave it to readers to make the obvious modification. Once and for all, we choose a homogeneous basis $\phi_{\alpha}$ $(\alpha=1, \ldots, \operatorname{dim} H)$ of $H$ with $\phi_{1}=1$. Let $\eta_{\mu \nu}=\left\langle\phi_{\mu}, \phi_{\nu}\right\rangle$ and $\left(\eta^{\mu \nu}\right)=\left(\eta_{\mu \nu}\right)^{-1}$.

Definition 3.0.3. A cohomological field theory is a collection of homomorphisms

$$
\Lambda_{g, k}: H^{\otimes k} \rightarrow H^{*}\left(\overline{\mathcal{M}}_{g, k}, \mathbb{Q}\right)
$$

satisfying the following properties:

C1. The element $\Lambda_{g, k}$ is invariant under the action of the symmetric group $S_{k}$.

C2. Let $g=g_{1}+g_{2}$ and $k=k_{1}+k_{2}$; Then the $\Lambda_{g, n}$ satisfy the composition property

$$
\begin{aligned}
& \rho_{\text {tree }}^{*} \Lambda_{g_{1}+g_{2}, k}\left(\alpha_{1}, \alpha_{2} \ldots, \alpha_{k}\right) \\
& \quad=\Lambda_{g_{1}, k_{1}+1}\left(\alpha_{i_{1}}, \ldots, \alpha_{i_{k_{1}}}, \mu\right) \eta^{\mu \nu} \otimes \Lambda_{g_{2}, k_{2}+1}\left(\nu, \alpha_{i_{k_{1}+1}}, \ldots, \alpha_{i_{k_{1}+k_{2}}}\right)
\end{aligned}
$$

for all $\alpha_{i} \in H$.

C3. Let

$$
\rho_{\text {loop }}: \overline{\mathcal{M}}_{g-1, k+2} \rightarrow \overline{\mathcal{M}}_{g, k}
$$


be the gluing loops morphism. Then

$$
\rho_{\text {loop }}^{*} \Lambda_{g, k}\left(\alpha_{1}, \alpha_{2}, \ldots, \alpha_{k}\right)=\Lambda_{g-1, k+2}\left(\alpha_{1}, \alpha_{2}, \ldots, \alpha_{n}, \mu, \nu\right) \eta^{\mu \nu},
$$

where $\alpha_{i}, \mu, \nu$, and $\eta$ are as in $\mathrm{C} 2$.

C4a. For all $\alpha_{i}$ in $H$ we have

$$
\Lambda_{g, k+1}\left(\alpha_{1}, \ldots, \alpha_{k}, 1\right)=\pi^{*} \Lambda_{g, k}\left(\alpha_{1}, \ldots, \alpha_{k}\right),
$$

C4b.

where $\pi: \overline{\mathcal{M}}_{g, n+1} \rightarrow \overline{\mathcal{M}}_{g, n}$ is the forgetful morphism.

$$
\int_{\overline{\mathcal{M}}_{0,3}} \Lambda_{0,3}\left(\alpha_{1}, \alpha_{2}, 1\right)=\left\langle\alpha_{1}, \alpha_{2}\right\rangle
$$

For each cohomological field theory, we can generalize the notion of intersection number, the generating function and total descendant potential function. Let

$$
\left\langle\tau_{l_{1}, \alpha_{1}}, \ldots, \tau_{l_{k}, \alpha_{k}}\right\rangle_{g}^{\Lambda}=\int_{\overline{\mathcal{M}}_{g, k}} \prod_{i} \psi_{i}^{l_{i}} \Lambda_{g, k}\left(\phi_{\alpha_{1}}, \ldots, \phi_{\alpha_{k}}\right) .
$$

Associating a formal variable $t_{i}^{\alpha}$ to $\tau_{i, \alpha}$, we define generating functions

$$
\mathcal{F}_{\Lambda}^{g}=\sum_{k \geq 0} \frac{t_{l_{1}}^{\alpha_{1}} \cdots t_{l_{k}}^{\alpha_{k}}}{k !}\left\langle\tau_{l_{1}, \alpha_{1}}, \ldots, \tau_{l_{k}, \alpha_{k}}\right\rangle_{g}
$$

and its total potential function

$$
\mathcal{D}_{\Lambda}=\exp \left(\sum_{g \geq 0} h^{g-1} \mathcal{F}_{\Lambda}^{g}\right) .
$$

Similarly, we can perform the dilaton shift

$$
q_{i}^{\alpha}= \begin{cases}t_{i}^{\alpha}, & t_{i}^{\alpha} \neq t_{1}^{1} \\ t_{1}^{1}-1, & \text { otherwise }\end{cases}
$$

The goal is to find other cohomological field theories such that their total descendant potential functions are $\tau$-functions of other integrable hierarchies.

\section{The theory of Fan-Jarvis-Ruan-Witten}

The classical integrable hierarchies are related to certain Dynkin diagrams. It is well-known that Dynkin diagrams naturally appear in singularity theory to describe the intersection pairing of its vanishing cycles. It has long been speculated in mathematics that the representation theory and singularity from the same Dynkin diagram should be related. This led to Witten's insight that the right place for the new geometry of integrable hierarchies is singularity theory. Singularity theory is a classical subject in mathematics. In the physical literature, it was referred as the Landau-Ginzburg model. In 
this section, we review Fan-Jarvis-Ruan's construction of "quantum" singularity theory based on Witten's remarkable PDE.

4.1. The singularity theory and its state spaces. In this section, we set up the cohomological data or state space of the theory. The input of the theory is a pair $(W, G)$ where $W$ is a "non-degenerate" quasihomogeneous polynomial $W: \mathbb{C}^{N} \rightarrow \mathbb{C}$ and $G$ is a group of abelian symmetries of $W$. Recall that $W$ is a quasihomogeneous polynomial if there is an $N$-tuple of rational numbers $\left(q_{1}, \ldots, q_{N}\right)$ such that

$$
W\left(\lambda^{q_{1}} x_{1}, \ldots, \lambda^{q_{N}} x_{N}\right)=\lambda W\left(x_{1}, \ldots, x_{N}\right)
$$

for $\lambda \in \mathbb{C}^{*} . q_{i}$ is called the degree or charge of $x_{i} . W$ is called non-degenerate if: (1) $W$ defines a unique singularity at zero; (2) the choice of $q_{i}$ is unique. A counterexample would be $W=x y$ where the choices of degree are not unique. To get ride of this example, we also assume that $W$ does not contain a term of the form $x y . g$ is called an abelian or diagonal symmetry of $W$ if $g$ is a diagonal matrix of the form $\operatorname{diag}\left(\lambda_{1}, \ldots, \lambda_{N}\right)$ such that

$$
W\left(\lambda_{1} x_{1}, \ldots, \lambda_{N} x_{N}\right)=W\left(x_{1}, \ldots, x_{N}\right) .
$$

We will use $G_{\max }$ to denote the full diagonal symmetry group. $G_{\max }$ is always nontrivial since it contains the element $J=\operatorname{diag}\left(e^{2 \pi i q_{1}}, \ldots, e^{2 \pi i q_{N}}\right)$. A certain subgroup $\langle J\rangle \subset G \subset G_{\max }$ plays an important role in the theory as well.

4.1.1. B-model. We start from the B-model theory which is more classical. The first classical B-model invariant is the local algebra (also known as the chiral ring or the Milnor ring)

$$
\mathcal{O}_{W}:=\mathbb{C}\left[x_{1}, \ldots, x_{N}\right] / \operatorname{Jac}(W),
$$

where $\operatorname{Jac}(W)$ is the Jacobian ideal generated by partial derivatives:

$$
J a c(W):=\left(\frac{\partial W}{\partial x_{1}}, \ldots, \frac{\partial W}{\partial x_{N}}\right)
$$

Then any symmetry group $G$ acts on the local algebra in the obvious way. But this is not the correct action. It is well-known that

$$
\mathcal{O}_{W} \cong \Omega^{N} / d W \wedge \Omega^{N-1},
$$

by

$$
\alpha \rightarrow \alpha d x_{1} \wedge d x_{2} \cdots \wedge d x_{N},
$$

where $\Omega^{i}$ is the space of holomorphic $i$-forms on $\mathbb{C}^{N}$. We will use the action of $G$ on $\Omega^{N} / d W \wedge \Omega^{N-1}$.

Let's review some of the basic facts about the local algebra. It is clear that the local algebra is generated by monomials. The degree of a monomial 
allows us to make the local algebra into a graded algebra. There is a unique highest-degree element $\operatorname{det}\left(\frac{\partial^{2} W}{\partial x_{i} \partial x_{j}}\right)$ with degree

$$
\hat{c}_{W}=\sum_{i}\left(1-2 q_{i}\right) .
$$

The degree $\hat{c}_{W}$ is called the central charge and is a fundamental invariant of the singularity $W$.

The singularities with $\hat{c}_{W}<1$ are called simple singularities and have been completely classified in the famous ADE-sequence. Quasi-homogeneous singularities of integral $\hat{c}_{W}$ with some additional conditions correspond to Calabi-Yau hypersurfaces in weighted projective space. Here, the singularity/LG-theory makes contact with Calabi-Yau geometry. There are many examples with fractional value $1 \leq \hat{c}_{w}$. These can be viewed as "fractional dimension Calabi-Yau manifolds" in a naive sense.

The dimension of the local algebra is given by the formula

$$
\mu=\prod_{i}\left(\frac{1}{q_{i}}-1\right) .
$$

Let $h_{i}$ be the dimension of the subspace of the local algebra of elements of degree $\lambda_{i}$. Its Poincaré polynomial $P(t, W)=\sum_{i} h_{i} t^{\lambda_{i}}$ can be computed explicitly. Recall that $d$ is the common denominator of the charges $q_{i}$ and that $q_{i}=\frac{n_{i}}{d}$. We have

$$
P\left(t^{d}, W\right)=\prod_{i=1}^{N} \frac{1-t^{d-n_{i}}}{1-t^{n_{i}}} .
$$

From the modern point of view, local algebra is considered to be part of the B-model theory of singularities. For its application, it is also important to orbifold the construction by $G$. The orbifold B-model graded vector space with pairing $\mathcal{O}_{W, G}$ was essentially worked out by the physicists IntriligatorVafa $[\mathbf{I V}]$ (see $[\mathbf{K a 1}]$ for a more mathematical account). The ring structure was constructed by Kaufmann-Krawitz [Ka1], $[\mathbf{K r}]$.

First of all, for each monomial in $\mathcal{O}_{W}$, we assign a double or Hodge grading $\left(d_{\mathbb{C}}, d_{\mathbb{C}}\right)$ where $d_{\mathbb{C}}$ is the degree of the monomial. For each $\gamma \in G$, let $\mathbb{C}_{\gamma}^{N}$ be the fixed points of $\gamma$ and $W_{\gamma}=\left.W\right|_{\mathbb{C}_{\gamma}^{N}}$. $W_{\gamma}$ is a quasihomogeneous singularity in fewer variables. Let $\gamma=\left(e^{2 \pi i \Theta_{1}^{\gamma}}, \ldots, e^{2 \pi i \Theta_{N}^{\gamma}}\right)$ where $\Theta_{i}^{\gamma} \in[0,1)$. Define

$$
\begin{aligned}
& \iota_{L}=\sum_{\Theta_{i}^{\gamma} \neq 0}\left(\Theta_{i}^{\gamma}-\frac{1}{2}\right)+\sum_{\Theta_{i}^{\gamma} \neq 0}\left(\frac{1}{2}-q_{i}\right) ; \\
& \iota_{R}=-\sum_{\Theta_{i}^{\gamma} \neq 0}\left(\Theta_{i}^{\gamma}-\frac{1}{2}\right)+\sum_{\Theta_{i}^{\gamma} \neq 0}\left(\frac{1}{2}-q_{i}\right) .
\end{aligned}
$$


We define

$$
\mathcal{O}_{\gamma}=\mathcal{O}_{W_{\gamma}}^{G}
$$

with the degree shifted by $\left(\iota_{L}, \iota_{R}\right)$. Namely, for any monomial $\alpha \in \mathcal{O}_{\gamma}$, we define

$$
\operatorname{deg}(\alpha)=\left(d_{\mathbb{C}}(\alpha)+\iota_{L}, d_{\mathbb{C}}(\alpha)+\iota_{R}\right)
$$

Define

$$
\mathcal{O}_{W, G}=\bigoplus_{\gamma \in G} \mathcal{O}_{\gamma}
$$

$\mathcal{O}_{W, G}$ is a doubly graded vector space. It is clearly a module over $\mathcal{O}_{W}^{G}$. Notice that $\mathcal{O}_{\gamma}$ is canonically isomorphic to $\mathcal{O}_{\gamma^{-1}}$. The pairing of $\mathcal{O}_{W, G}$ is the direct sum of residue pairings

$$
\langle,\rangle: \mathcal{O}_{\gamma} \otimes \mathcal{O}_{\gamma^{-1}} \rightarrow \mathbb{C}
$$

via the above canonical isomorphism.

The main problem is to construct an associative multiplication.

DeFinition 4.1.1. Let $W=\sum_{j}^{T} c_{j} W_{j}$ be a nondegenerate quasihomogeneous polynomial, where $W_{j}$ are monomials. $W$ is called invertible if $T=N$.

The notion of invertible singularity first appeared in the physics literature $[\mathbf{B H}]$ and is extremely important in mirror symmetry. An important result due to Kreuzer $[\mathbf{K R}]$ is that $W$ is the sum of three types of basic invertible singularities

Fermat: $x^{i}$.

Loop: $x_{1}^{a_{1}} x_{2}+x_{2}^{a_{2}} x_{3}+\cdots+x_{n}^{a_{n}} x_{1}$.

Chain: $x_{1}^{a_{1}} x_{2}+x_{2}^{a_{2}} x_{3}+\cdots+x_{n}^{a_{n}}$.

For invertible singularities, Kaufmann-Krawitz defined an associative multiplication as follows. We define a product on $\bigoplus_{\gamma} \mathcal{O}_{W_{\gamma}}$ and then take invariants. The product has the properties

$$
\mathcal{O}_{W_{\gamma_{1}}} \otimes \mathcal{O}_{W_{\gamma_{2}}} \rightarrow \mathcal{O}_{W_{\gamma_{1} \gamma_{2}}}
$$

as well as respecting the $\mathcal{O}_{W}$-module structure in the sense that

$$
\alpha 1_{g_{1}} \star \beta 1_{g_{2}}=\alpha \beta 1_{g_{1}} \star 1_{g_{2}},
$$

where $\alpha, \beta \in \mathcal{O}_{W_{1}}$ and $1_{g}$ is the unity in $\mathcal{O}_{W_{g}}$. Let

$$
1_{g_{1}} \star 1_{g_{2}}=\gamma_{g_{1}, g_{2}} 1_{g_{1} g_{2}} .
$$

Then,

$$
\gamma_{g_{1}, g_{2}}= \begin{cases}0, & \text { there exists } z_{i} \text { such that } g_{1} z_{i} \neq z_{i}, \\ & g_{2} z_{i} \neq z_{i}, g_{1} g_{2} z_{i} \neq z_{i} \\ \operatorname{Hess}\left(\left.W\right|_{\mathbb{C}^{I}}\right), & i \in I \text { iff } g_{1} z_{i} \neq z_{i}, g_{2} z_{i} \neq z_{i}, g_{1} g_{2} z_{i}=z_{i}\end{cases}
$$

where Hess means the Hessian and we use the convention that HessW $\left.\right|_{\{0\}}=1$. 
THEOREM 4.1.2. (Kaufmann-krawitz) For invertible $W$ and $G \subset S L \cap$ $G_{\text {max }}$, i.e., matrix of determinant one, $\star$ is associative with unit $1 \in \mathcal{O}_{W}$ and invariant under $G$. Furthermore, it preserves the double or Hodge grading.

By taking invariants, we obtain a Frobenius algebra $\mathcal{O}_{W, G}$ for an invertible polynomial and $G \subset S L \cap G_{\max }$.

Notice that the miniversal deformation space $D(W)$ of $W$ has a structure of Frobenius manifold. A major problem of the subject is to orbifold this Frobenius manifold in the sense of Kauffman-Krawitz's orbifolding Frobenius algebra $\mathcal{O}_{W, G}$.

4.1.2. A-model. The A-model state space was introduced by Fan-JarvisRuan [FJR1, FJR2, FJR3] as part of the moduli theory of the Witten equation. It is closely related to the theory of vanishing cycles, Lefschetz thimbles, and Picard-Lefschetz theory.

Consider the Milnor fibration $W: \mathbb{C}^{N} \rightarrow \mathbb{C}$. It is an honest fiber bundle away from critical values. Fix a base point $z_{0}$ with $\left|z_{0}\right| \gg 0$. The fiber $W^{-1}\left(z_{0}\right)$ is a Stein manifold, homotopy equivalent to a bouquet of middledimensional spheres. Therefore, its reduced homology vanishes, except in the middle dimension. There is a geometric way to describe these homology classes as follows. Suppose that $U$ is the miniversal deformation space of the singularity. Topologically, $U$ is a ball in $\mathbb{C}^{\mu}$. Incidentally, the local algebra can be interpreted as the tangent space of $U$. We extend the Milnor fibration to $F: U \times \mathbb{C}^{N} \rightarrow U \times \mathbb{C}$ as $F(\lambda, x)=\left(\lambda, F_{\lambda}(x)\right)$, where $F_{\lambda}(x)$ is the miniversal deformation of $W$, i.e., $F(0, x)=W$. Here, everything is in the germ sense, and we can assume that the critical values of $F$ are in $U \times D_{M}$ for a large disc $D_{M}$. We can also assume that $z_{0} \notin D_{M}$, so $F_{\lambda}^{-1}\left(z_{0}\right) \rightarrow U$ is a fiber bundle. Hence, $H_{N-1}\left(F_{\lambda}^{-1}\left(z_{0}\right)\right)$ forms a vector bundle. Furthermore, the integral homology defines a lattice and hence a Gauss-Manin connection. The Gauss-Manin connection is characterized by the property that the covariant constant sections are precisely the sections of integral homology classes. We use a section of an integral homology class, or Gauss-Manin connection, to trivialize the vector bundle and identify the class of $W^{-1}\left(z_{0}\right)$ as a flat section of the middle dimension homological bundle.

For a generic value of $\lambda$, the function $F_{\lambda}$ is a holomorphic Morse function. A distinguished basis of $H_{N-1}\left(F_{\lambda}^{-1}\left(z_{0}\right)\right)$ can be constructed from a system of paths $u_{i}:[0,1] \rightarrow \mathbb{C}$ connecting $z_{0}$ to the critical values where $\mathbb{C} \cong\{\lambda\} \times$ $\mathbb{C} \subset U \times \mathbb{C}$. A system of paths connecting $z_{0}$ to critical values $z_{i}$ is called distinguished if

(i) $u_{i}$ has no self-intersection,

(ii) $u_{i}, u_{j}$ has no intersection except $u_{i}(0)=u_{j}(0)=z_{0}$, and

(iii) the paths $u_{1}, \ldots, u_{\mu}$ are numbered in the same order in which they enter the point $z_{0}$, counter-clockwise.

For each $u_{i}$, we can associate a homology class $\delta_{i} \in H_{N-1}\left(F_{\lambda}^{-1}\left(z_{0}\right)\right)$ as a vanishing cycle along $u_{i}$. More precisely, the neighborhood of the critical 
point of $z_{i}$ contains a local vanishing cycle. Then $\delta_{i}$ is obtained by transporting the local vanishing cycle to $z_{0}$ using the homotopy lifting property. The cycle $\delta_{i}$ is unique up to homotopy of $u_{i}$ as long as the homotopy does not pass through another critical value. Then, $\delta_{1}, \ldots, \delta_{\mu}$ defines a distinguished basis of $H_{N-1}\left(F_{\lambda}^{-1}\left(z_{0}\right)\right)$. When we deform $\lambda$, the critical values of $F_{\lambda}$ deform. One can deform the system of paths homotopically. The corresponding vanishing cycles define an integral section of the middle dimensional homological bundle, and hence a parallel transport from $H_{N-1}\left(F_{\lambda}^{-1}\left(z_{0}\right)\right)$ to $H_{N-1}\left(F_{\lambda^{\prime}}^{-1}\left(z_{0}\right)\right)$.

The choice of distinguished system of paths is not unique. They are related by Picard-Lefschetz transformations. One can associate a loop $\beta_{i}$ going along the path $u_{i}$ from the point $z_{0}$ to the point $z_{i}$, going round the point $z_{i}$ in the positive direction (counter-clockwise) and returning along the path $u_{i}$ to the point $z_{0}$. We can use $\beta_{i}$ to change a path $u_{j}$ to $u_{j}^{\prime}$ by following $\beta_{i}$ first and then $u_{j}$. The effect on the vanishing cycle is denoted by $h_{\beta_{i}}\left(\delta_{j}\right)$, where

$$
h_{\beta_{i}}\left(\delta_{j}\right)=\delta_{j}+(-1)^{N(N+1) / 2}\left(\delta_{i} \circ \delta_{j}\right) \delta_{i},
$$

where $\delta_{i} \circ \delta_{j}$ is the intersection number. This is called a Picard-Lefschetz transformation. It turns out that any two systems of distinguished paths can be transformed into each other by a sequence of Picard-Lefschetz transformations.

The most important classical invariant of vanishing cycles is the intersection matrix $\Gamma_{W}=\left(\delta_{i} \circ \delta_{j}\right)$. Choose a basis. One can draw a Dynkin diagram $\Gamma_{W}$ for the intersection matrix. It is well-known that the Dynkin diagram of ADE-singularities is an ADE-Dynkin diagram. This is where singularity theory makes contact with infinite dimensional Lie algebra. It is still an open question how to construct an infinite dimensional Lie algebras and its integrable representations for a Dynkin diagram of a general singularity. When we say integrable hierarchy of $W$, we mean the integrable hierarchy corresponding to $\Gamma_{W}$. In general, the intersection matrix is not necessarily non-degenerate.

Consider the exact sequence of relative homology

$$
H_{N}\left(\mathbb{C}^{N}, \mathbb{Z}\right) \rightarrow H_{N}\left(\mathbb{C}^{N}, F_{\lambda}^{-1}\left(z_{0}\right), \mathbb{Z}\right) \stackrel{\partial}{\rightarrow} H_{N-1}\left(F_{\lambda}^{-1}\left(z_{0}\right), \mathbb{Z}\right) \rightarrow H_{N-1}\left(\mathbb{C}^{N}, \mathbb{Z}\right) .
$$

Using this sequence, it is easy to see that $\partial$ is an isomorphism and has rank $\mu$. Furthermore, the isomorphism can also be understood geometrically. Let $u_{i}$ be a path as defined previously. We define the Lefschetz thimble $\Delta_{i}$ as the union of vanishing cycles along the path $u_{i}$. It is clear that $\partial \Delta_{i}=\delta_{i}$ and hence defines a relative homology class. The boundary map $\partial$ gives the above connecting homomorphism. It is clear that the Picard-Lefschetz theory is trivially adapted to the basis $\Delta_{1}, \ldots, \Delta_{\mu}$.

It is convenient for us to push the base point $z_{0}$ to $-\infty$ by considering $H_{N}\left(\mathbb{C}^{N},\left(\operatorname{Re} F_{\lambda}\right)^{-1}(-\infty,-M), \mathbb{Z}\right)$ for $M \gg 0$. To simplify the notation, we denote $\left(\operatorname{Re} F_{\lambda}\right)^{-1}(-\infty, M)\left(\operatorname{resp} .\left(\operatorname{Re} F_{\lambda}\right)^{-1}(M, \infty)\right)$ for $M \gg 0$ by $F_{\lambda}^{-\infty}$ 
(resp. $F_{\lambda}^{\infty}$ ). In this case, we consider virtually horizontal paths extending from a critical value and becoming a horizontal path eventually.

The Lefschetz thimble along a virtually horizontal path $\gamma$ defines an integral class $\Delta_{\gamma} \in H_{N}\left(\mathbb{C}^{N}, F_{\lambda}^{-\infty}, \mathbb{Z}\right)$ A particular important case is when $F_{\lambda}$ is strongly regular in the sense that the critical values have distinct imaginary parts. The horizontal paths give a canonical choice and hence define a distinguished basis of the middle dimension homology. By abusing notation, we call the relative homology $H_{N}\left(\mathbb{C}^{N}, F^{-\infty}, \mathbb{Z}\right)$ the space of Lefschetz thimbles. A theorem of Wall [Wa1, Wa2] shows that the dual space of Lefschetz thimbles (as well as the space of vanishing cycles) is isomorphic to the local algebra as a $G$-space.

The main advantage of considering Lefschetz thimbles is the fact that the intersection pairing

$$
\left.H_{N}\left(\mathbb{C}^{N}, F_{\lambda}^{\infty}, \mathbb{Z}\right) \otimes H_{N}\left(\mathbb{C}^{N}, F_{\lambda}^{-\infty}, \infty\right), \mathbb{Z}\right) \rightarrow \mathbb{Z}
$$

is perfect, whereas the intersection pairing for vanishing cycles may be degenerate. The pairing for Lefschetz thimbles is well-defined for all $\lambda$ and is preserved by parallel transport via the Gauss-Manin connection. It is easy to check that the pairing for holomorphic Morse functions is given by the intersection of stable with unstable manifolds of critical points. Therefore, it is perfect.

The above pairing induces a pairing on relative cohomology

$$
H^{N}\left(\mathbb{C}^{N}, F_{\lambda}^{-\infty}, \mathbb{C}\right) \otimes H^{N}\left(\mathbb{C}^{N}, F_{\lambda}^{\infty}, \mathbb{C}\right) \rightarrow \mathbb{C}
$$

as follows. Suppose that $\left\{\Delta_{1}, \ldots, \Delta_{\mu}\right\}$ is a basis of $H_{N}\left(\mathbb{C}^{N}, F_{\lambda}^{-\infty}, \mathbb{Z}\right)$, and $\left\{\tilde{\Delta}_{1}, \ldots, \tilde{\Delta}_{\mu}\right\}$ is a basis of $H_{N}\left(\mathbb{C}^{N}, F_{\lambda}^{+\infty}, \mathbb{Z}\right)$. Let $\Delta^{*}, \tilde{\Delta}^{*}$ be the dual bases. We define the intersection matrix

$$
\left(\left\langle\Delta_{i}^{*}, \tilde{\Delta}_{j}^{*}\right\rangle\right)=\left(\left\langle\Delta_{i}, \tilde{\Delta}_{j}\right\rangle\right)^{-1}
$$

Then, we define

$$
\langle\alpha, \beta\rangle=\sum_{\Delta_{i}, \tilde{\Delta}_{j}} \alpha\left(\Delta_{i}\right) \beta\left(\tilde{\Delta}_{j}\right)\left\langle\Delta_{i}^{*}, \tilde{\Delta}_{j}^{*}\right\rangle .
$$

We remark that the above pairing is not the wedge product, even if $\alpha$ and $\beta$ are viewed as differential forms.

Back to our situation of $W$ (i.e., $\lambda=0$ ), we wish to consider the following pairing. As we have done before, write $q_{i}=n_{i} / d$ for a common denominator $d$, and choose $\xi$ such that $\xi^{d}=-1$. Multiplication by the diagonal matrix $\left(\xi^{n_{1}}, \ldots, \xi^{n_{N}}\right)$ defines a map

$$
I: \mathbb{C}^{N} \rightarrow \mathbb{C}^{N}
$$

sending $W^{ \pm \infty} \rightarrow W^{\mp \infty}$. Hence, it induces an isomorphism

$$
I_{*}: H_{N}\left(\mathbb{C}^{N}, W^{\mp \infty}, \mathbb{C}\right) \rightarrow H_{N}\left(\mathbb{C}^{N}, W^{ \pm \infty}, \mathbb{C}\right) .
$$


Definition 4.1.3. We define a pairing on $H_{N}\left(\mathbb{C}^{N}, W^{ \pm \infty}, \mathbb{Z}\right)$ by

$$
\left\langle\Delta_{i}, \Delta_{j}\right\rangle=\left\langle\Delta_{i}, I_{*}\left(\Delta_{j}\right)\right\rangle \text {. }
$$

It induces a pairing $\langle\alpha, \beta\rangle=\left\langle\alpha, I^{*}(\beta)\right\rangle$ on $H^{N}\left(\mathbb{C}^{N}, W^{ \pm \infty}, \mathbb{C}\right)$. As noted earlier, changing the choice of $\xi$ will change the isomorphism $I$ by an element of the group $\langle J\rangle$, and $I^{2} \in\langle J\rangle$. Therefore, the pairing is independent of the choice of $I$ on the invariant subspace $H_{N}\left(\mathbb{C}^{N}, W^{\infty}, \mathbb{Z}\right)^{\langle J\rangle}$.

Now we shall "orbifold" the previous construction.

Definition 4.1.4. We define the $\gamma$-twisted sector $\mathcal{H}_{\gamma}$ of the state space to be the $G$-invariant part of the middle-dimensional relative cohomology for $W_{\gamma}$. That is,

$$
\mathcal{H}_{\gamma}:=H^{N_{\gamma}}\left(\mathbb{C}_{\gamma}^{N}, W_{\gamma}^{\infty}, \mathbb{Q}\right)^{G},
$$

with its intersection pairing, where $N_{\gamma}$ is the dimension of $\mathbb{C}_{\gamma}^{N}$. The central charge for the singularity $W_{\gamma}$ is

$$
\hat{c}_{\gamma}:=\sum_{i: \Theta_{i}^{\gamma}=0}\left(1-2 q_{i}\right) .
$$

As in Chen-Ruan orbifold cohomology theory, we should shift the degree.

Definition 4.1.5. Suppose that $\gamma=\left(e^{2 \pi i \Theta_{1}^{\gamma}}, \ldots, e^{2 \pi i \Theta_{N}^{\gamma}}\right)$ for rational numbers $0 \leq \Theta_{i}^{\gamma}<1$.

We define the degree shifting number

$$
\begin{aligned}
\iota_{\gamma} & =\sum_{i}\left(\Theta_{i}^{\gamma}-q_{i}\right) \\
& =\frac{\hat{c}_{W}-N_{\gamma}}{2}+\sum_{i: \Theta_{i}^{\gamma} \neq 0}\left(\Theta_{i}^{\gamma}-1 / 2\right) \\
& =\frac{\hat{c}_{\gamma}-N_{\gamma}}{2}+\sum_{i: \Theta_{i}^{\gamma} \neq 0}\left(\Theta_{i}^{\gamma}-q_{i}\right) .
\end{aligned}
$$

For a class $\alpha \in \mathcal{H}_{\gamma}$, we define

$$
\operatorname{deg}_{W}(\alpha)=\operatorname{deg}(\alpha)+2 \iota_{\gamma} .
$$

Proposition 4.1.6. For any $\gamma \in G_{W}$ we have

$$
\iota_{\gamma}+\iota_{\gamma^{-1}}=\hat{c}_{W}-N_{\gamma},
$$

and for any $\alpha \in \mathcal{H}_{\gamma}$ and $\beta \in \mathcal{H}_{\gamma^{-1}}$ we have

$$
\operatorname{deg}_{W}(\alpha)+\operatorname{deg}_{W}(\beta)=2 \hat{c}_{W} .
$$

REMARK 4.1.7. $H^{N}\left(\mathbb{C}^{N}, W^{-1}\left(z_{0}\right), \mathbb{C}\right)$ also carries an internal Hodge grading due to its mixed Hodge structure. This defines a bi-grading for $\mathcal{H}_{\gamma}$ with double shifts $\left(\iota_{\gamma}, \iota_{\gamma}\right)$. 
Definition 4.1.8. The quantum cohomology group or A-model state space of the singularity $W / G$ is defined [FJR2] as

$$
\mathcal{H}_{W, G}=\bigoplus_{\gamma \in G} \mathcal{H}_{\gamma}
$$

Definition 4.1.9. The $J$-sector $\mathcal{H}_{J}$ is always one-dimensional, and the constant function 1 defines a generator $\in \mathcal{H}_{J}$ of degree 0 . This element is the unit in the ring $\mathcal{H}_{W, G}$.

Definition 4.1.10. For any $\gamma \in G$, we say that the $\gamma$-sector is NeveuSchwarz if the fixed point locus is trivial (i.e., $N_{\gamma}=0$ ). If the fixed point locus is non-trivial, we say that the $\gamma$-sector is Ramond.

Since $\gamma$ and $\gamma^{-1}$ have the same fixed point set, there is an obvious isomorphism

$$
\varepsilon: \mathcal{H}_{\gamma} \rightarrow \mathcal{H}_{\gamma^{-1}}
$$

We define a pairing on $\mathcal{H}_{W}$ as the direct sum of pairings

$$
\langle,\rangle_{\gamma}: \mathcal{H}_{\gamma} \otimes \mathcal{H}_{\gamma^{-1}} \rightarrow \mathbb{C}
$$

as $\langle f, g\rangle_{\gamma}=\left\langle f, \varepsilon^{*} g\right\rangle$, where the second pairing is the pairing of the space of relative cohomology. The above pairing is obviously symmetric and nondegenerate.

Lemma 4.1.11. The above pairing preserves the degree. Namely, it is a pairing of $\mathcal{H}_{W, G}^{a}$ with $\mathcal{H}_{W, G}^{2 \hat{c}-a}$ :

$$
\mathcal{H}_{W, G}^{a} \otimes \mathcal{H}_{W, G}^{2 \hat{W}^{-a}} \rightarrow \mathbb{C} .
$$

Remark 4.1.12. The lemma indicates that one can view $W / G$ as an object of complex dimension $\hat{c}_{W}$. Under the shift, $\mathcal{H}_{J}$ has degree 0 . On the other hand, the non-twisted sector has degree $\hat{c}_{W}$, and the sector $\mathcal{H}_{J^{-1}}$ has degree $2 \hat{c}_{W}$. We have not yet defined the multiplication on $\mathcal{H}_{W, G}$ which is part of the quantization theory of Fan-Jarvis-Ruan-Witten (see next section).

\subsection{Witten equation and quantization of singularity theory.} We have introduced the state space of a singularity for the A-model. Next, we will construct the cohomological field theory $\Lambda_{g, k}^{W, G}$ for the A-model state space. It can be viewed as the quantization of A-model singularity theory. This was accomplished by Fan-Jarvis-Ruan. The central problem is to solve the following Witten equation for any quasihomogeneous singularity. All the information such as state space described in the last section is the natural outcome of a solution to this problem. 
The Witten equation is a system of nonlinear elliptic PDE associated to a quasihomogeneous polynomial $W$. Formally, it has the simple form

$$
\bar{\partial} u_{i}+\frac{\overline{\partial W}}{\partial u_{i}}=0
$$

where $W$ is a quasi-homogeneous polynomial, and $u_{i}$ is interpreted as a section of an appropriate orbifold line bundle $L_{i}$ on a Riemann surface $C$. Some simple examples are

$$
\begin{aligned}
& \left(A_{r} \text {-case }\right): \bar{\partial} u+\bar{u}^{r}=0 \\
& \left(D_{n} \text {-case }\right): \bar{\partial} u_{1}+n \bar{u}_{1}^{n-1}+\bar{u}_{2}^{2}=0, \bar{\partial} u_{2}+2 \bar{u}_{1} \bar{u}_{2}=0 . \\
& \left(E_{7} \text {-case }\right): \bar{\partial} u_{1}+3 \bar{u}_{1}^{2}+\bar{u}_{2}^{3}=0, \bar{\partial} u_{2}+3 \bar{u}_{1} \bar{u}_{2}^{2}=0 .
\end{aligned}
$$

For the Witten equation to make sense, $L_{i}$ is required to have some special properties. This can be summarized as the following interesting moduli problem in algebraic geometry. It can be thought of as background data for the Witten equation.

4.2.1. Moduli space of $W$-structures. Let's first set up some notation. $C$ denotes a marked nodal stable Riemann surface. $L_{i}$ is an orbifold line bundle associated to the variable $z_{i}$. Without loss of generality, we assume that all the orbifold points of $L_{i}$ are marked or nodal points.

$$
K_{l o g, C}=K_{C} \otimes O\left(x_{1}\right) \otimes \cdots \otimes O\left(x_{k}\right)
$$

where $x_{1}, \ldots, x_{k}$ are all the marked points.

Definition 4.2.1. Suppose that $W=\sum_{j=1}^{s} c_{j} W_{j}$ where $W_{j}=\prod_{i=1}^{N} z_{i}^{b_{i j}}$ is a monomial. For any non-degenerate, quasi-homogeneous polynomial $W \in$ $\mathbb{C}\left[x_{1}, \ldots, x_{N}\right]$, we define a $W$-structure to be the data of an $N$-tuple $\left(L_{1}, \ldots, L_{N}\right)$ of orbifold line bundles on $C$ and isomorphisms $\varphi_{j}: W_{j}\left(L_{1}, \ldots\right.$, $\left.L_{N}\right) \rightarrow K_{C, \log }$ for every $j \in\{1, \ldots, s\}$, where

$$
W_{j}\left(L_{1}, \ldots, L_{N}\right)=L_{1}^{\otimes b_{1, j}} \otimes \cdots \otimes L_{N}^{\otimes b_{N, j}} .
$$

Without the loss of generality, we assume that the orbifold structure only happens at marked or nodal points.

Definition 4.2.2. Given any two $W$-structures $\mathbb{L}:=\left(L_{1}, \ldots, L_{N}, \varphi_{1}, \ldots\right.$, $\left.\varphi_{s}\right)$ and $\mathbb{L}^{\prime}:=\left(L_{1}^{\prime}, \ldots, L_{N}^{\prime}, \varphi_{1}^{\prime}, \ldots \varphi_{s}^{\prime}\right)$ on $C$, it is clear that a set of morphisms $\xi_{j}: L_{j} \rightarrow L_{j}^{\prime}$ of orbifold line bundles for $j \in\{1, \ldots, N\}$ will induce a morphism $\Xi_{l}: W_{l}\left(L_{1}, \ldots, L_{N}\right) \rightarrow W_{l}\left(L_{1}^{\prime}, \ldots, L_{N}^{\prime}\right)$ for every $l \in\{1, \ldots, s\}$.

An isomorphism of $W$-structures $\Upsilon: \mathbb{L} \rightarrow \mathbb{L}^{\prime}$ on $C$ is defined to be a collection of isomorphisms $\xi_{j}: L_{j} \rightarrow L_{j}^{\prime}$ such that for every $l \in\{1, \ldots, s\}$ we have $\varphi_{l}=\varphi_{l}^{\prime} \circ \Xi_{l}$.

Definition 4.2.3. We denote the stack of stable $W$-orbicurves by $\mathcal{W}_{g, k}$. 
Note that for each marked point $x_{j} \in C$, an orbifold line bundle $L$ at $x_{j}$ is given by the local orbifold chart

$$
\left.L_{i}\right|_{\Delta / \mathbb{Z} / m \mathbb{Z}} \cong(\Delta \times \mathbb{C}) / \mathbb{Z} / m \mathbb{Z},
$$

where $\Delta$ is a disc and the generator $e \in \mathbb{Z} / m \mathbb{Z}$ acts as $e(z, s)=(\exp (2 \pi i / m) z$, $\left.\exp \left(2 \pi i v_{i} / m\right) s\right)$. We choose the same $m$ for each $L_{i}$. Let $\gamma=\operatorname{diag}\left(\exp \left(2 \pi i v_{1} /\right.\right.$ $\left.m), \ldots, \exp \left(2 \pi i v_{k} / m\right)\right)$. We further requires that $\langle\gamma\rangle$ acts on $\mathbb{C}^{N}$ effectively. It is rather amazing that

Lemma 4.2.4. $\gamma \in G_{\max }$. Namely, $\gamma$ defines an automorphism of $W$.

The upshot of the lemma is that the orbifold structure of $\bigoplus_{i} L_{i}$ is completely parameterized by the automorphisms of $W$. Therefore, the group $G_{\max }$ enters the theory in a natural way. One may wonder if we can insist that $L_{i}$ is an honest line bundle. Then, there will be no $W$-structure in general. Even if it exists sometimes, its degeneration on a nodal Riemann surface will naturally introduce an orbifold structure at nodes. In short, there is no way one can avoid the orbifold structure. The theory of Fan-Jarvis-RuanWitten naturally corresponds to the orbifolded singularity $W / G_{\max }$. It is possible to generalize it to $W / G$ for $\langle J\rangle \subset G$ (see the discussion of admissible groups). The current theory does not work for any group smaller than $\langle J\rangle$ in any generality.

Definition 4.2.5. A marked point $x_{j}$ of a $W$-curve is called NeveuSchwarz if the fixed point locus $\mathbb{C}_{\gamma}^{N} \subseteq \mathbb{C}^{N}$ is just $\{0\}$. The point $x_{j}$ is called Ramond otherwise and the variable $z_{i} \in \mathbb{C}_{\gamma}^{N}$ is called a Ramond variable.

Another classical construction is to desingularize the orbifold structure. Namely, one can change the local trivialization of $L_{i}$ by

$$
(z, s) \rightarrow\left(z^{m}, z^{v_{i}} s\right)
$$

Under the new local trivialization, $L_{i}-\left(\left.L_{i}\right|_{x_{j}}\right)$ extends to an orbifold line bundle $\left|L_{i}\right|\left(x_{j}\right)$ smooth over $x_{j}$. Furthermore,

$$
H^{0}\left(C, L_{i}\right)=H^{0}\left(C,\left|L_{i}\right|\left(x_{j}\right)\right), H^{1}\left(C, L_{i}\right)=H^{1}\left(C,\left|L_{i}\right|\left(x_{j}\right)\right) .
$$

After we desingularize all the orbifold structure, we denote it by $\left|L_{i}\right|$.

There are several natural morphisms analogous to that of $\overline{\mathcal{M}}_{g, k}$.

Stabilization. Forgetting the $W$-structure and the orbifold structure gives a morphism

$$
\text { st }: \mathcal{W}_{g, k} \rightarrow \overline{\mathcal{M}}_{g, k}
$$

THEOREM 4.2.6. $\mathcal{W}_{g, k}$ is a compact smooth complex orbifold and st is a finite map. 
Components. $\mathcal{W}_{g, k}$ has many components. It is easy to show that the orbifold structure at each marked point remains the same for each component.

Definition 4.2.7. For any choice $\gamma:=\left(\gamma_{1}, \ldots, \gamma_{k}\right) \in G_{\max }^{k}$ we define $\mathcal{W}_{g, k}(\gamma)$ to be the open and closed substack of $\mathcal{W}_{g, k}$ with the given orbifold structure specified by $\gamma$.

We define the type of any $W$-structure in $\mathcal{W}_{g, k}(\gamma)$ to be $\gamma \in G_{\text {max }}^{k}$.

There is an important selection rule that must be satisfied for $\mathcal{W}_{g, k}(\gamma)$ to be non-empty. Although the degree of an orbifold bundle $L_{i}$ on $C$ may be a rational number, the degree of $\left|L_{i}\right|$ must be an integer, so for all $i \in\{1, \ldots, s\}$ the following equations must hold for integral values of $\operatorname{deg}\left(\left|L_{j}\right|\right)$ :

$$
\sum_{j=1}^{N} b_{j i} \operatorname{deg}\left(\left|L_{j}\right|\right)=2 g-2+k-\sum_{l=1}^{k} \sum_{j=1}^{N} b_{j i} \Theta_{j}^{\gamma_{l}} .
$$

Moreover, because $W$ is non-degenerate, the weights $q_{j}$ are uniquely determined by the requirement that they satisfy the equations $\sum_{j=1}^{N} b_{j i} q_{j}=1$ for all $i \in\{1, \ldots, s\}$, so we find that for every $j \in\{1, \ldots, N\}$ we have

$$
\operatorname{deg}\left(\left|L_{j}\right|\right)=\left(q_{j}(2 g-2+k)-\sum_{l=1}^{k} \Theta_{j}^{\gamma_{l}}\right) \in \mathbb{Z} .
$$

Forgetful morphism. As we mentioned earlier, we can desingularize the orbifold structure at a marked point $x_{j}$ and obtain orbifold line bundles $\left|L_{i}\right|\left(x_{j}\right)$. But $\left|L_{1}\right|\left(x_{j}\right), \ldots,\left|L_{N}\right|\left(x_{j}\right)$ is not a $\mathrm{W}$-structure in general except that the orbifold structure at $x_{j}$ is $J$.

If $\gamma=\left(\gamma_{1}, \ldots, \ldots, J\right)$, then we can forget the last marked point in the same way as the forgetful morphism for $\overline{\mathcal{M}}_{g, k}$. More precisely, If $\left(L_{1}, \ldots, L_{N}\right)$ is a $W$-structure with the above orbifold decorations. $\left(\left|L_{1}\right|\left(x_{k}\right), \ldots,\left|L_{N}\right|\left(x_{k}\right)\right)$ is a $W$-structure on $C$ with $x_{k}$ being forgotten. The reason that this happens is the following calculation:

$$
\begin{aligned}
W_{i}\left(\left|L_{1}\right|\left(x_{k}\right), \ldots,\left|L_{N}\right|\left(x_{k}\right)\right) & \rightarrow K_{C, \log } \otimes \mathbb{O}\left(\left(-\sum_{j=1}^{N} b_{i j} \Theta_{j}^{J}\right) x_{k}\right) \\
& =K_{C, \log } \otimes \mathbb{O}\left(\left(-\sum_{j=1}^{N} b_{i j} q_{j}\right) x_{k}\right) \\
& =K_{C, \log } \otimes \mathbb{O}\left(-x_{k}\right) \\
& =K_{C^{\prime}, \log },
\end{aligned}
$$

where $C^{\prime}$ is the marked Riemann surface obtained from $C$ by forgetting $x_{k}$.

Therefore, we define a morphism

$$
\pi: \mathcal{W}_{g, k}\left(\gamma_{1}, \ldots, \gamma_{k-1}, J\right) \rightarrow \mathcal{W}_{g, k-1}\left(\gamma_{1}, \ldots, \gamma_{k-1}\right)
$$


We call it the forgetful morphism.

Note that the essential property of $\gamma_{i}$ that allows the forgetful morphism to exist is the fact that $\sum_{j=1}^{N} b_{l j} \Theta_{j}^{\gamma_{i}}=1$ for every $l \in\{1, \ldots, s\}$. Since the weights $q_{j}$ are uniquely determined by $W$, this means that a marked point $x_{i}$ may not be forgotten unless $\gamma_{i}=J$.

EXAMPLE 4.2.8. For the three-pointed, genus-zero $W$-structure, the choice of orbifold line bundles $L_{1}, \ldots, L_{N}$ providing the $W$-structure is unique, if it exists at all. If it exists, then $\mathcal{W}_{0,3}(\gamma)$ is isomorphic to $B G_{\max }$.

Unfortunately, there is no direct lift of $\rho_{\text {tree }}, \rho_{\text {loop }}$ from page 9 to the moduli stack of $W$-curves because there is no canonical way to glue the fibers of the line bundles $L_{i}$ on the two points that map to a node.

But we could describe a gluing process in terms of an additional structure that we call rigidification. Suppose the fiber of the $W$-structure at the marked point $p$ is $\left[\left(L_{1} \oplus L_{2} \oplus \cdots \oplus L_{N}\right) / G_{p}\right]$, where $G_{p}$ is the local group. The rigidification can be thought as a $G_{p}$-equivariant map $\psi: \bigoplus_{i} L_{i} \rightarrow \mathbb{C}^{N}$ commuting with the $W$-structure. For any element $g \in G_{p}$, the rigidification $g \psi$ is considered to be an equivalent rigidification.

Alternatively, $\psi$ is equivalent to a choice of basis $e_{i} \in L_{i}$ such that $W_{j}\left(e_{1}, \ldots, e_{N}\right)=d z / z$ and the basis $g\left(e_{1}\right), \ldots, g\left(e_{N}\right)$ is considered to be an equivalent choice. In particular, if $L_{i_{1}}, \ldots, L_{i_{m}}$ are the line bundles fixed by $G_{p}$ (we call the corresponding variables $z_{i_{j}}$ the Ramond variables) then in each equivalance class of rigidification, the basis elements $e_{i_{1}}, \ldots, e_{i_{m}}$ for the subspace $\left.\bigoplus_{j=1}^{m} L_{i_{j}}\right|_{p}$ are unique, but the basis elements for the terms not fixed by $G_{p}$ (the Neveu-Schwarz variables) are only unique up to the action of $G_{p}$.

It is clear that the group $G_{\max } / G_{p}$ acts transitively on the set of rigidifications within a single orbit. Let $\mathcal{W}_{g, k}^{r i g_{p}}$ be the equivalence classes of $W$-curves with a rigidification at $p$. The group $G_{\max } / G_{p}$ acts on $\mathcal{W}_{g, k}^{r i g_{p}}$ by interchanging the rigidifications. It is clear that $\mathcal{W}_{g, k}^{r i g_{p}}$ is a principal $G_{\text {max }} / G_{p}$-bundle over $\mathcal{W}_{g, k}$. We use $\mathcal{W}_{g, k}^{\text {rig }}$ to denote the moduli stack of rigidified $W$-structures at all the marked points.

Now we describe the gluing. To simplify notation, we ignore the orbifold structures at other marked points and denote the types of the marked points $p_{+}, p_{-}$being glued by $\gamma_{+}, \gamma_{-}$. Recall that the resulting orbifold structures must be balanced, which means that $\gamma_{-}=\gamma_{+}^{-1}$. Let

$$
\psi_{ \pm}: j_{p_{ \pm}}^{*}\left(L_{1} \oplus \cdots \oplus L_{N}\right) \rightarrow\left[\mathbb{C}^{N} / G_{p_{ \pm}}\right]
$$

be the rigidifications. However, the residues at $p_{+}, p_{-}$are opposite to each other. The obvious identification will not preserve the rigidifications. Here, we fix once and for all an isomorphism

$$
I: \mathbb{C}^{N} \rightarrow \mathbb{C}^{N}
$$


such that $W(I(z))=-W(z)$. I has been explicitly constructed previously as follows. Suppose that $q_{i}=n_{i} / d$ for the common denominator $d$. Choose $\xi^{d}=-1$. Then,

$$
I\left(z_{1}, \ldots, z_{N}\right)=\left(\xi^{n_{1}} z_{1}, \ldots, \xi^{n_{N}} z_{N}\right) .
$$

The identification by $I$ induces a $W$-structure on the nodal orbifold Riemann surface with a rigidification at the nodal point. Forgetting the rigidification at the node yields the lifted gluing morphisms

$$
\begin{aligned}
& \tilde{\rho}_{\text {tree }, \gamma}: \mathcal{W}_{g_{1}, k_{1}+1}^{\text {rig }}(\gamma) \times \mathcal{W}_{g_{2}, k_{2}+1}^{\text {rig }}\left(\gamma^{-1}\right) \rightarrow \mathcal{W}_{g_{1}+g_{2}, k_{1}+k_{2}}, \\
& \widetilde{\rho}_{\text {loop }, \gamma}: \\
& \mathcal{W}_{g, k+2}^{\text {rig }}\left(\gamma, \gamma^{-1}\right) \rightarrow \mathcal{W}_{g+1, k} .
\end{aligned}
$$

4.2.2. Virtual cycle from Witten equation. A casual investigation of the Witten equation reveals that the Witten equation is much more subtle than its simple appearance would suggest. Suppose that $u_{i} \in \Omega^{0}\left(L_{i}\right)$. A simple computation shows

$$
\bar{\partial} u_{i} \in \Omega^{0,1}\left(L_{i}\right), \frac{\overline{\partial W}}{\partial u_{i}} \in \Omega_{\log }^{0,1}\left(\bar{L}_{i}^{-1}\right),
$$

where log means a $(0,1)$-form with possible singularities of order $\leq 1$. Namely, the Witten equation has singular coefficients! This is a fundamental phenomenon for the application of the Witten equation. One of the most difficult conceptual advances in the entire theory was to generate the A-model state space from the study of the Witten equation. Now it is understood that the singularity of the Witten equation is the key. Unfortunately, the appearance of singularities makes the Witten equation very difficult to study analytically. In fact, it has taken the authors six years to construct the theory!

Another subtle issue is the fact that we need an isomorphism $\bar{L}_{i}^{-1} \cong L_{i}$ for the two terms of the Witten equation living in the same space. The required isomorphism can be obtained by a choice of metric. A nontrivial fact is that such a metric can be constructed uniformly from a metric of the underlying Riemann surface. Then the question is: which metric should we choose on the Riemann surface? We should mention that a different choice of metric often leads to a completely different looking theory, including a different dimension for its moduli space. Apparently, there is no physical guidance for the correct metric we should choose. The authors have experimented with both smooth and cylindrical metrics near marked points. Now we understand that both choices are important for the theory!

Let's briefly outline the construction. Let $\mathcal{W}_{g, k}\left(\gamma_{1}, \ldots, \gamma_{k}\right)$ be the moduli space of $W$-structures decorated by the orbifold structure $\gamma_{i}$ at the marked point $x_{i}$. It can be considered as the background data to set up the Witten equation. Unfortunately, it is rather difficult to solve the Witten equation due to the fact that $W$ is highly degenerate. It is much easier to solve a perturbed equation for $W+W_{0}$, where $W_{0}$ is a linear perturbation term such that $W_{\gamma}+W_{0 \gamma}$ is a holomorphic Morse function for every $\gamma$. Here $W_{\gamma}, W_{0 \gamma}$ are the restrictions of $W, W_{0}$ to the fixed point set $\mathbb{C}_{\gamma}^{N}$. The background data 
for perturbed Witten equation is the moduli space of rigidified $W$-structures $\mathcal{W}_{g, k}^{r i g}\left(\gamma_{1}, \ldots, \gamma_{k}\right)$.

The crucial part of the analysis is to show that a solution of the Witten equation converges to a critical point of $W_{\gamma_{i}}+W_{0 \gamma_{i}}$. This enables us to construct a moduli space

$$
\mathcal{W}_{g, k}^{s}\left(\kappa_{j_{1}}, \ldots, \kappa_{j_{k}}\right)
$$

of solutions of the perturbed Witten equation converging to the critical point $\kappa_{j_{i}}$ at the marked point $x_{i}$. We call $W_{0}$ strongly regular if (i) $W_{\gamma_{i}}+W_{0 \gamma_{i}}$ is holomorphic Morse; (ii) the critical values of $W_{\gamma_{i}}+W_{0 \gamma_{i}}$ have distinct imaginary parts. The first important result is

THEOREM 4.2.9. If $W_{0}$ is strongly regular, then $\mathcal{W}_{g, k}^{s}\left(\kappa_{j_{1}}, \ldots, \kappa_{j_{k}}\right)$ is compact and has a virtual fundamental cycle $\left[\mathcal{W}_{g, k}^{s}\left(\kappa_{j_{1}}, \ldots, \kappa_{j_{k}}\right)\right]^{\text {vir }}$ of degree

$$
2\left(\left(c_{W}-3\right)(1-g)+k-\sum_{i} \iota_{\gamma_{i}}\right)-\sum_{i} N_{\gamma_{i}} .
$$

Here, $\iota_{\gamma_{i}}$ is the degree shifting number defined previously.

It turns out to be convenient to map the above virtual cycle into $H_{*}\left(\mathcal{W}_{g, k}^{\text {rig }}, \mathbb{Q}\right)$ even though it is not a subspace of the latter in any way. This is the first step of the construction.

We have not yet seen the state space. Then, a crucial new phenomenon comes into play when we study how the above virtual cycle changes when we vary the perturbation. It turns out that the above virtual cycle does depend on the perturbation. It will change when $W_{0}$ fails to be strongly regular. Recall that for a strongly regular perturbation we can construct a canonical system of HORIZONTAL paths $u_{i}^{ \pm}$'s and the associated Lefschetz thimble $\Delta_{i}^{ \pm}$. When we perturb $W_{0}$ crossing the "wall" (where the imaginary parts of critical values happen to be the same), we arrive at another canonical system of paths and its Lefschetz thimble $\Delta_{i}^{\prime \pm}$. The relation between $\Delta_{i}^{ \pm}$ and $\Delta_{i}^{\prime \pm}$ is determined by well-known Picard-Lefschetz formula. The "wall crossing formula" for virtual fundamental cycles can be summarized in the following quantum Picard-Lefschetz theorem:

THEOREM 4.2.10. When $W_{0}$ varies, $\left[\mathcal{W}_{g, k}^{s}\left(\kappa_{j_{1}}, \ldots, \kappa_{j_{k}}\right)\right]^{\text {vir }}$ transforms in the same way as the Lefschetz thimble $\Delta_{j_{i}}^{-}$attached to the critical point $k_{j_{i}}$.

$\Delta_{i}^{+}$'s transform in the opposite way as $\Delta_{i}^{-}$'s. It is well-known that the "diagonal class" $\sum_{i} \Delta_{i}^{-} \otimes \Delta_{i}^{+}$is independent of perturbation. It suggests the following definition of an "extended virtual class". To simplify the notation, we assume that there is only one marked point with the orbifold decoration $\gamma$. Then, the wall crossing formula of $\left[\mathcal{W}_{g, 1}^{s}\left(\kappa_{i}\right)\right]^{v i r}$ shows precisely that

$$
\sum_{j}\left[\mathcal{W}_{g, 1}^{s}\left(\kappa_{j}\right)\right]^{v i r} \otimes \Delta_{j}^{+}
$$


viewed as a class in $H_{*}\left(\mathcal{W}_{g, 1}^{r i g}(\gamma), \mathbb{Q}\right) \otimes H_{N_{\gamma}}\left(\mathbb{C}_{\gamma}^{N_{\gamma}}, W_{\gamma}^{\infty}, \mathbb{Q}\right)$ is independent of the perturbation. Now, we define

$$
\left[\mathcal{W}_{g, 1}^{s}(\gamma)\right]^{v i r}=\sum_{j}\left[\mathcal{W}_{g, 1}^{s}\left(\kappa_{j}\right)\right]^{v i r} \otimes \Delta_{j}^{+}
$$

The above definition can be generalized to multiple marked points in an obvious way. It is obvious that

$$
\left[\mathcal{W}_{g, k}^{s}\left(\gamma_{1}, \ldots, \gamma_{k}\right)\right]^{v i r} \in H_{*}\left(\mathcal{W}_{g, k}^{r i g}\left(\gamma_{1}, \ldots, \gamma_{k}\right), \mathbb{Q}\right) \otimes \prod_{i} H_{N_{\gamma_{i}}}\left(\mathbb{C}^{N_{\gamma_{i}}}, W_{\gamma_{i}}^{\infty}, \mathbb{Q}\right)
$$

of degree

$$
2\left(\left(c_{W}-3\right)(1-g)+k-\sum_{i} \iota \gamma_{i}\right)
$$

COROLlaRY 4.2.11. $\left[\mathcal{W}_{g, k}^{s}\left(\gamma_{1}, \ldots, \gamma_{k}\right)\right]^{\text {vir }}$ is independent of the perturbation $W_{0}$.

$W_{0}$ is only part of the perturbation data. Eventually, we want to work on $\mathcal{W}_{g, k}$. It is known that so: $\mathcal{W}_{g, k}^{s} \rightarrow \mathcal{W}_{g, k}$ is a quasi-finite proper map by forgetting all the rigidifications. We can define

$$
\left[\mathcal{W}_{g, k}\left(\gamma_{1}, \ldots, \gamma_{k}\right)\right]^{v i r}:=\frac{(-1)^{\chi}}{\operatorname{deg}(s o)}(s o)_{*}\left[\mathcal{W}_{g, k, W}^{s}\left(\gamma_{1}, \ldots, \gamma_{k}\right)\right]^{v i r}
$$

where

$$
\chi=c_{W}(1-g)+k-\sum_{i} \iota_{\gamma_{i}} .
$$

The independence of the above virtual cycle on rigidification implies that

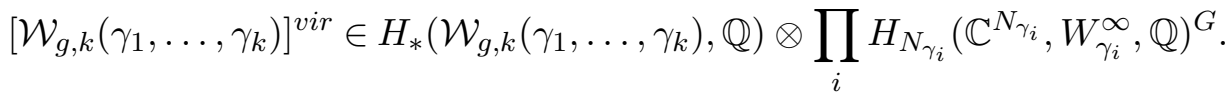

One gets a cleaner formula by pushing down $\left[\mathcal{W}_{g, k}(\gamma)\right]^{\text {vir }}$ to $\overline{\mathcal{M}}_{g, k}$.

Definition 4.2.12. Let $\Lambda_{g, k}^{W} \in \operatorname{hom}\left(\mathcal{H}_{W}^{\otimes k}, H^{*}\left(\overline{\mathcal{M}}_{g, k}\right)\right)$ be given for homogeneous elements $\left(\alpha_{1}, \ldots, \alpha_{k}\right)$ with $\alpha_{i} \in \mathcal{H}_{\gamma_{i}}$ by

$$
\Lambda_{g, k}^{W}\left(\alpha_{1}, \ldots, \alpha_{k}\right):=\frac{\left|G_{\max }\right|^{g}}{\operatorname{deg}(s t)} P D s t_{*}\left(\left[\mathcal{W}_{g, k}(\gamma)\right]^{v i r} \cap \prod_{i=1}^{k} \alpha_{i}\right)
$$

and then extends linearly to general elements of $\mathcal{H}_{W}^{\otimes k}$.

Let 1 be the distinguished generator of $\mathcal{H}_{J}$, and let $\langle,\rangle^{W}$ denote the pairing on the state space $\mathcal{H}_{W}$. 
TheOrem 4.2.13. The collection $\left(\mathcal{H}_{W, G_{\max }},\langle,\rangle^{W},\left\{\Lambda_{g, k}^{W}\right\}, 1\right)$ is a cohomological field theory.

Moreover, if $W_{1}$ and $W_{2}$ are two singularities in distinct variables, then the cohomological field theory from $W_{1}+W_{2}$ is the tensor product of the cohomological field theories arising from $W_{1}$ and $W_{2}$ :

$$
\left(\mathcal{H}_{W_{1}+W_{2}, G_{\max }},\left\{\Lambda_{g, k}^{W_{1}+W_{2}}\right\}\right)=\left(\mathcal{H}_{W_{1}, G_{\max }} \otimes \mathcal{H}_{W_{2}, G_{\max }},\left\{\Lambda_{g, k}^{W_{1}} \otimes \Lambda_{g, k}^{W_{2}}\right\}\right) .
$$

4.3. Admissible group. So far, we are working with the group $G_{\max }$. It is also useful to generalize it to other groups. The group $\langle J\rangle$ is particularly important in application.

Definition 4.3.1. A subgroup $G \subset G_{\max }$ is called admissible if there is a quasihomogeneous Laurent series $Z$ of the same quasihomogeneity such that $G=G_{\max }(W+Z)$.

Let $(\mathcal{W}+\mathcal{Z})_{g, k}$ be the moduli space of $W$-structures defined by $W+Z$. It is an open closed substack of $\mathcal{W}_{g, k}$. One can show that $(\mathcal{W}+\mathcal{Z})_{g, k}$ is independent of $Z$ and we denote it by $\mathcal{W}_{g, k, G}$. It has the property that all the orbifold decorations are from $G$. In essence, we just use $Z$ to single out an open closed substack of $\mathcal{W}_{g, k}$ satisfying all the formal properties of $\mathcal{W}_{g, k}$ with orbifold decoration only from $G$. It was shown in [FJR2] that $\langle J\rangle$ is admissible. Furthermore, Krawitz proved that any group $G$ containing $J$ is admissible. Furthermore, an explicit description of $\mathcal{W}_{g, k,\langle J\rangle}$ has been worked out in [CR1].

One can simply restrict the virtual class to $\mathcal{W}_{g, k, G}$ and define a cohomological field theory $\Lambda_{g, k, G}^{W}$ as well as $\mathcal{F}_{W, G}^{g}, \mathcal{D}_{W, G}$.

\section{ADE-hierarchies and Witten conjecture}

The main motivation for Witten to introduce his equation is the following conjecture.

ConjeCture 5.0.2 (ADE-Integrable Hierarchy Conjecture). : The total potential functions of the $A, D$, and $E$ singularities with group $\langle J\rangle$ are $\tau$ functions of the corresponding $A, D$, and $E$ integrable hierarchies.

The $A_{n}$-case has been established recently by Faber-Shadrin-Zvonkin [FSZ]. One of the main applications of the theory of Fan-Jarvis-RuanWitten is to verify Witten's integrable hierarchies conjecture for the $D E$ series. It turns out that Witten's conjecture needs a modification in the $D_{n}$ case for $n$ odd. This modification is extremely interesting because it reveals a surprising role that mirror symmetry plays in integrable hierarchies.

Let's start from the ADE-hierarchies. As we mentioned in section two, there are two versions of ADE-integrable hierarchies - that of DrinfeldSokolov $[\mathbf{D S}]$ and that of Kac-Wakimoto $[\mathbf{K W}]$. Both of these were constructed from the basic representation of affine Kac-Moody algebras. They 
are equivalent by $[\mathbf{H M}]$. The version directly relevant to us is the KacWakimoto ADE-hierarchies because the following beautiful work of FrenkelGivental-Milanov reduces the problem to an explicit problem in GromovWitten theory! Let's describe their work.

Let $W$ be a nondegenerate quasihomogeneous singularity and $\phi_{i}(1 \leq$ $i \leq \mu$ ) be the monomial basis of the Milnor ring with $\phi_{1}=1$. Consider the miniversal deformation space $\mathbb{C}^{\mu}$ where a point $\lambda=\left(t_{1}, \ldots, t_{\mu}\right)$ parameterizes the polynomial $W+t_{1} \phi_{1}+t_{2} \phi_{2} \cdots+t_{\mu} \phi_{\mu}$. We can assign a degree to $t_{i}$ such that the above perturbed polynomial has the degree one, i.e., $\operatorname{deg}\left(t_{i}\right)=1-\operatorname{deg}\left(\phi_{i}\right)$. The tangent space $T_{\lambda}$ carries an associative multiplication $\circ$ and an Euler vector field $E=\sum_{i} \operatorname{deg}\left(t_{i}\right) \partial_{t_{i}}$ with the unit $e=\frac{\partial W_{\lambda}}{\partial t_{1}}$. It is more subtle to construct a metric. We can consider residue pairing

$$
\langle f, g\rangle_{\lambda}=\operatorname{Res}_{x=0} \frac{f g \omega}{\frac{\partial W_{\lambda}}{\partial x_{1}} \cdots \frac{\partial W_{\lambda}}{\partial x_{N}}}
$$

using a holomorphic $n$-form $\omega$. A deep theorem of Saito $[\mathbf{S}]$ states that one can choose a primitive form $\omega$ such that the induced metric is flat. Together, it defines a Frobenius manifold structure on a neighborhood of zero of $\mathbb{C}^{\mu}$. We should mention that there is no explicit formula of primitive form in general. However, it is known that for ADE-singularities the primitive can be chosen as a constant multiple of standard volume form, i.e., $c d x$ for $A_{n}$ and $c d x d y$ for DE-series.

Furthermore, one can define a potential function $\mathcal{F}$ playing the role of genus zero Gromov-Witten theory with only primary fields. It is constructed as follows. We want to work in flat coordinates $s_{i}$ with the property that $\operatorname{deg}_{\mathbb{C}}\left(s_{i}\right)=\operatorname{deg}_{\mathbb{C}}\left(t_{i}\right)$ and $\left\langle\partial_{s_{i}}, \partial_{s_{j}}\right\rangle$ are constant. The flat coordinates depend on the flat connection of metric and hence the primitive form. Its calculation is important and yet a difficult problem. Nevertheless, we know that the flat coordinates exist thanks to the work of Saito $[\mathbf{S}]$. Then, consider the 3point correlator $C_{i j k}=\left\langle\partial_{s_{i}}, \partial_{s_{j}}, \partial_{s_{k}}\right\rangle$ as a function near zero in $\mathbb{C}^{\mu}$. We can integrate $C_{i j k}$ to obtain $\mathcal{F}$. Here, we normalize $\mathcal{F}$ such that $\mathcal{F}$ has the degree three leading term. We can differentiate $\mathcal{F}$ by the Euler vector field. It has the property $L_{E} \mathcal{F}=\left(\hat{c}_{W}-3\right) \mathcal{F}$. Namely, $\mathcal{F}$ has homogeneous degree $\hat{c}_{W}-3$. The last condition means that, in the Taylor expansion

$$
\begin{gathered}
\mathcal{F}=\sum a\left(n_{1}, \ldots, n_{\mu}\right) \frac{s_{1}^{n_{1}} \cdots s_{\mu}^{n_{\mu}}}{n_{1} ! \cdots n_{\mu} !} \\
a\left(n_{1}, \ldots, n_{\mu}\right) \neq 0
\end{gathered}
$$

only when $\sum n_{i}-\sum n_{i}\left(1-\operatorname{deg}_{\mathbb{C}}\left(s_{i}\right)\right)=\sum \operatorname{deg}_{\mathbb{C}}\left(s_{i}\right)=\hat{c}_{W}-3$. Notes that the degree in Frobenius manifold is different from that of the $A$-model. For example, the unit $e$ has the degree 1 instead of zero. The $A$-model degree is $1-$ the $\mathrm{B}$-model degree. With this relation in the mind, we will treat the insertion $s_{i}$ with degree $1-\operatorname{deg}_{\mathbb{C}}\left(s_{i}\right)$. Then, the above formula is precisely the selection rule of quantum singularity theory. 
It is known that the Frobenius manifold of a singularity is semisimple in the sense that the Frobenius algebra on $T_{\lambda}$ at a generic point $\lambda$ is semisimple. On any semisimple Frobenius manifold, Givental constructed a formal Gromov-Witten potential function. We will only be interested in the case that the Frobenius manifold is the one corresponding to the miniversal deformation space of a quasihomogenous singularity $W$. We denote it by

$$
\mathcal{D}_{W, \text { formal }}=\exp \left(\sum_{g \geq 0} h^{2 g-2} \mathcal{F}_{\text {formal }}^{g}\right) \text {. }
$$

The construction of $\mathcal{D}_{W, \text { formal }}$ is complicated. We only need its following formal properties

(1) $\mathcal{F}_{\text {formal }}^{0}$ agree with $\mathcal{F}$ for primary field, i.e., no descendants.

(2) The coefficients of $\mathcal{F}_{\text {formal }}^{g}$ behaves like the correlators of a GromovWitten theory with $C_{1}=0$ and dimension $\hat{c}_{W}$.

(3) $\mathcal{D}_{W, \text { formal }}$ satisfies all the formal axioms of Gromov-Witten theory. The first property is obvious from the construction. The second property is the consequence of the fact that $\mathcal{D}_{W, \text { formal }}$ satisfies dilaton equation and Virasoro constraint. A fundamental theorem of Frenkel-Givental-Milanov $[\mathbf{G M}],[\mathbf{F G M}]$ is

Theorem 5.0.3. For ADE-singularities, $\mathcal{D}_{W, \text { formal }}$ is a $\tau$-function of the Kac-Wakimoto ADE-hierarchies.

The main theorem is

\section{THEOREM 5.0.4.}

(1) Except for $D_{n}$ ( $n$ odd), the total potential functions of DE-singularities with the group $\langle J\rangle$ are equal to the corresponding Givental formal Gromov-Witten potential functions for a linear change of variables.

$$
\mathcal{D}_{D_{n}, G_{\text {max }}}=\mathcal{D}_{A_{2 n-3}, \text { formal }},
$$

up to a linear change of variables.

(3) For $D_{n}^{T}=x^{n-1} y+y^{2}(n \geq 4), \mathcal{D}_{D_{n}^{T}, G_{m a x}}=\mathcal{D}_{D_{n}, \text { formal }}$, up to a linear change of variables.

Using the theorem of Frenkel-Givental-Milanov, we obtain

Corollary 5.0.5. (1) Except for $D_{n}$ ( $n$ odd), the total potential function of DE-singularities with the group $\langle J\rangle$ is a $\tau$-function of the corresponding Kac-Wakimoto hierarchies (and hence DrinfeldSokolov hierarchies).

(2) The total potential function of $D_{n}$-singularities with the maximal diagonal symmetry group is a $\tau$-function of the $A_{2 n-3}$ Kac-Wakimoto hierarchies (and hence Drinfeld-Sokolov hierarchies). 
(3) The total potential function of $D_{n}^{T}=x^{n-1} y+y^{2} \quad(n \geq 4)$ with the maximal diagonal symmetry group is a $\tau$-function of the $D_{n}$ KacWakimoto hierarchies (and hence Drinfeld-Sokolov hierarchies).

The proof of the main theorem depends on three key ingredients. The first ingredient is a strong reconstruction theorem of ADE-theory to reduce the calculation to that of genus zero, 3-point correlators or certain explicit 4-point correlators. The second ingredient is Topological Euler class axioms for Neveu-Schwarz sectors which enable us to compute all the 3-point and required 4-point correlators. The last ingredient is to understand the mirror symmetry of ADE-singularities, which is the subject of another conjecture of Witten. The required modification in the $D_{n}$ case will become transparent once we understand the mirror symmetry.

REMARK 5.0.6. There is a technical issue in Givental's formal theory, as follows. For any semisimple point $t$ of Saito's Frobenius manifold, he defined an ancestor potential $\mathcal{A}_{t}$. From this he obtains a descendant potential function $\mathcal{D}=\hat{S}_{t} \mathcal{A}_{t}$, where $\hat{S}_{t}$ is certain quantization of a symplectic transformation $S_{t}$ determined by the Frobenius manifold. Then, he showed $\mathcal{D}$ is independent of $t$. However, to compare with our $A$-model calculation, we need to expand $\mathcal{D}$ as formal power series at $t=0$. Although $\mathcal{D}$ is expected to have a power series expansion at $t=0$, we have been informed that a proof is not yet in the literature. Our strategy to avoid this problem is to show that (i) the A- and B-models have isomorphic Frobenius manifolds, and (ii) in the $\mathrm{ADE}$ cases the ancestor (as well as descendant) functions of both models are completely determined by their respective Frobenius manifolds. Therefore, the A- and B-model have the same ancestor potentials and hence the same descendant potentials.

5.1. Reconstruction theorem. In general, it is difficult to compute the full GW-invariants. But there is a physical reason to believe that singularity theory is much more accessible to computation. This is particularly strong in the ADE-case. The key idea is that there is a rather strong reconstruction theorem for ADE-singularities to reduce the computations to 3point correlators and certain specific 4-point correlators. In this subsection, we will outline the reconstruction theorem simultaneously for ADE-quantum singularity theory and Givental's formal Gromov-Witten theory in the ADE case. We use the fact that (i) both theories satisfy the formal axioms of Gromov-Witten theories; (ii) both have the same selection rules; (iii) both have isomorphic quantum rings up to a mirror transformation. The last fact will be established at the end of this section. To simplify the notation, we state the theorem for Givental's formal Gromov-Witten theory. It is understood that the same argument applies to the quantum singularity theory of the A-model mirror.

We start with the higher genus reconstruction using an idea of FaberShadrin-Zvonkin [FSZ]. 
THEOREM 5.1.1. If $\hat{c}_{W}<1$, then the total descendant or ancestor potential function is uniquely determined by the genus-zero primary potential (i.e., without gravitational descendants). If $\hat{c}_{W}=1$, then the potential function is uniquely determined by its genus-zero and genus-one primary potentials.

The proof of Theorem 5.1.1 is a direct consequence of the following two lemmas, using the Faber-Shadrin-Zvonkine reduction technique. For this argument we always assume that $\hat{c}_{W} \leq 1$.

LemMA 5.1.2. Let $\alpha_{i} \in \mathcal{H}_{\gamma_{i}, G}$ for all $i \in\{1, \ldots, k\}$ and let $\beta$ be any product of $\psi$-classes. If $\hat{c}_{W}<1$, then the integral $\int_{\overline{\mathcal{M}}_{g, k}} \beta \Lambda_{g, k}^{W, G}\left(\alpha_{1}, \ldots, \alpha_{k}\right)$ vanishes if $\operatorname{deg}(\beta)<g$ for $g \geq 1$. If $\hat{c}=1$, then the above integral vanishes when $\operatorname{deg}(\beta)<g$ for $g \geq 2$.

Lemma 5.1.3. Suppose that $\beta$ is the product of $\psi_{i}$ classes. If $\operatorname{deg}(\beta) \geq g$, then $\beta$ is supported on the boundary of $\overline{\mathcal{M}}_{g, k}$.

REMARK 5.1.4. There is an alternative higher-genus reconstruction, using Teleman's recent announcement [ $\mathbf{T e}]$ of a proof of Givental's conjecture [Gi4]. However, the ADE-case is so simple that the above argument achieves the same goal.

Next, we consider the reconstruction of genus-zero correlators using WDVV.

Definition 5.1.5. We call a class $\gamma$ primitive if it cannot be written as $\gamma=\gamma_{1} \star \gamma_{2}$ for $0<\operatorname{deg}_{\mathbb{C}}\left(\gamma_{i}\right)<\operatorname{deg}_{\mathbb{C}}(\gamma)$ (or, in the case of our A-model singularity theory $\left.0<\operatorname{deg}_{W}\left(\gamma_{i}\right)<\operatorname{deg}_{W}(\gamma)\right)$.

We have the following lemma.

Lemma 5.1.6 (Reconstruction Lemma). Any genus-zero k-point correlator of the form

$$
\left\langle\gamma_{1}, \ldots, \gamma_{k-3}, \alpha, \beta, \epsilon \star \phi\right\rangle
$$

can be rewritten as

$$
\begin{aligned}
\left\langle\gamma_{1}, \ldots, \gamma_{k-3}, \alpha, \beta, \epsilon \star \phi\right\rangle= & S+\left\langle\gamma_{1}, \ldots, \gamma_{k-3}, \alpha, \epsilon, \beta \star \phi\right\rangle \\
& +\left\langle\gamma_{1}, \ldots, \gamma_{k-3}, \alpha \star \epsilon, \beta, \phi\right\rangle \\
& -\left\langle\gamma_{1}, \ldots, \gamma_{k-3}, \alpha \star \beta, \epsilon, \phi\right\rangle,
\end{aligned}
$$

where $S$ is a linear combination of genus-zero correlators with fewer than $k$ insertions.

Moreover, all the genus-zero $k$-point correlators $\left\langle\gamma_{1}, \ldots, \gamma_{k}\right\rangle$ are uniquely determined by the pairing, by the 3-point correlators, and by correlators of the form $\left\langle\alpha_{1}, \ldots, \alpha_{k^{\prime}-2}, \alpha_{k^{\prime}-1}, \alpha_{k^{\prime}}\right\rangle$ for $k^{\prime} \leq k$, and such that $\alpha_{i}$ primitive for all $i \leq k^{\prime}-2$. 
DeFINITION 5.1.7. We call a correlator a basic correlator if it is of the form described in the previous lemma, that is, if all insertions are primitive except for the last two.

For a basic correlator $\left\langle a_{1}, \ldots, a_{k}\right\rangle$, we still have the dimension formula

$$
\sum_{i} \operatorname{deg}_{\mathbb{C}}\left(a_{i}\right)=\hat{c}_{W}+k-3 .
$$

This gives the following corollary.

COROllary 5.1.8. If $\operatorname{deg}_{\mathbb{C}}(a) \leq \hat{c}_{W}$ for all classes $a$ and if $P$ is the maximum complex degree of any primitive class, then all the genus-zero correlators are uniquely determined by the pairing and $k$-point correlators with

$$
k \leq 2+\frac{1+\hat{c}_{W}}{1-P}
$$

In particular, $k \leq 4$ for all the simple singularities in the (A-model) theory. For the $D_{n+1}^{T}$ case in the (A-model) theory we have $k \leq 5$. On the B-model side, we have $k \leq 4$ for the $A_{n}$-series and $E_{6}, E_{7}$ and $E_{8}$ as well as $D_{n+1}^{T}$, while we have $k \leq 5$ for the $D_{n+1}$ series.

To set up the notation for the next reconstruction lemma, we recall

$$
\begin{gathered}
A_{n}=\frac{x^{n+1}}{n+1}, D_{n}=x^{n-1}+x y^{2}, D_{n}^{T}=x^{n-1} y+y^{2}, \\
E_{6}=x^{3}+y^{4}, E_{7}=x^{3}+x y^{3}, E_{8}=x^{3}+y^{5} .
\end{gathered}
$$

Their local algebras $\mathcal{O}_{W}$ are generated by the monomials in the variables $X, Y$. The Reconstruction Lemma yields more detailed information for the basic correlators as well.

\section{THEOREM 5.1.9.}

(1) All genus-zero correlators in the $A_{n-1}$ case for both the (A-model) and the Saito (B-model) theory are uniquely determined by the pairing, the 3-point correlators and a single 4-point correlator of the form $\left\langle X, X, X^{n-2}, X^{n-2}\right\rangle$.

(2) All genus-zero correlators in the $D_{n+1}$ case of the (A-model) theory with maximal symmetry group, and in the $D_{n+1}^{T}$ case of the Saito (B-model), are uniquely determined by the pairing, the 3-point correlators, and a single 4-point correlator of the form $\left\langle X, X, X^{2 n-2}\right.$, $\left.X^{2 n-2}\right\rangle$.

(3) All genus-zero correlators in the $D_{n+1}^{T}$ case of the (A-model) theory, in the $D_{n+1}$ case of the theory with $n$ odd and symmetry group $\langle J\rangle$, and in the $D_{n+1}$ case of the Saito (B-model) are uniquely determined by the pairing, the 3-point correlators, and 4-point correlators of the form $\left\langle X, X, X^{n-1}, X^{n-2}\right\rangle$. 
(4) In the $E_{6}$ case of theory with maximal symmetry group, and in the $E_{6} B$-model, all genus-zero correlators are uniquely determined by the pairing, the 3-point correlators, and the correlators $<\left\langle Y, Y, Y^{2}\right.$, $\left.X Y^{2}\right\rangle$ and $\langle X, X, X Y, X Y\rangle$.

(5) In the $E_{7}$-case (both $A$ - and $B$-model), all genus-zero correlators are uniquely determined by the pairing, the 3-point correlators, and the correlators $\left\langle X, X, X^{2}, X Y\right\rangle,\left\langle X, Y, X^{2}, X^{2}\right\rangle,\left\langle X, Y, Y^{2}, X^{2} Y\right\rangle$, and $\left\langle Y, Y, X Y, X^{2} Y\right\rangle$.

(6) In the $E_{8}$-case of our theory with maximal symmetry group, and in the $E_{8} B$-model, all genus-zero correlators are uniquely determined by the pairing, the 3-point correlators, and by the correlators $\left\langle Y, Y, Y^{3}, X Y^{3}\right\rangle$, and $\left\langle X, X, X, X Y^{3}\right\rangle$.

5.2. Calculations in the Neveu-Schwarz sector. The remaining task is to calculate the 3-point and above 4-point correlators explicitly and match them. On the B-model side, we need to calculate flat coordinates of a Frobenius manifold and expand the potential function in flat coordinates. In order to do this, we need an explicit form of the primitive form which could be problematic in general. Fortunately, the primitive form is known to be the standard volume $c d x d y$ form for an ADE-singularity. In fact, all the genus-zero correlators have been calculated already in both mathematics $[\mathbf{N Y}]$ and physics.

The problem is mostly on the A-side. An explicit calculation of A-model correlators requires counting the number of solutions of the Witten equation. At this point, this is out of reach except for the case of the Neveu-Schwarz sector. Recall that $\gamma \in G$ is called Neveu-Schwarz if $\mathbb{C}_{\gamma}^{N}=\{0\}$, i.e., no fixed point except zero. In the set-up of a smooth metric at the marked point (smooth theory), it was a lemma of Witten that the unperturbed Witten equation has only the zero solution. This leads to the following.

Suppose that all the decorations are Neveu-Schwarz, meaning that $\mathbb{C}_{\gamma_{i}}^{N}=$ $\{0\}$, and so we can omit $H^{N_{\gamma_{i}}}\left(\mathbb{C}_{\gamma_{i}}^{N}, W_{\gamma_{i}}^{\infty}, \mathbb{Q}\right)=\mathbb{C}$ from our notation (that is, we assume that all the marked points are decorated with the element $\left.1 \in H^{N_{\gamma_{i}}}\left(\mathbb{C}_{\gamma_{i}}^{N}, W_{\gamma_{i}}^{\infty}, \mathbb{Q}\right)\right)$.

Consider the universal $W$-structure $\left(\mathcal{L}_{1}, \ldots, \mathcal{L}_{N}\right)$ on the universal curve $\pi: \mathcal{C} \rightarrow \mathcal{W}_{g, k, G}\left(\gamma_{1}, \ldots, \gamma_{k}\right)$ and the two-term complex of sheaves

$$
\pi_{*}\left(\left|\mathcal{L}_{i}\right|\right) \rightarrow R^{1} \pi_{*}\left(\left|\mathcal{L}_{i}\right|\right)
$$

There is a family of maps

$$
W_{i}=\frac{\partial W}{\partial x_{i}}: \pi_{*}\left(\bigoplus_{j}\left|\mathcal{L}_{j}\right|\right) \rightarrow \pi_{*}\left(K \otimes\left|\mathcal{L}_{i}\right|^{*}\right) \cong R^{1} \pi_{*}\left(\left|\mathcal{L}_{i}\right|\right)^{*}
$$


The above two-term complex is quasi-isomorphic to a complex of vector bundles $[\mathbf{P V}]$

$$
E_{i}^{0} \stackrel{d_{i}}{\rightarrow} E_{i}^{1}
$$

such that

$$
\operatorname{ker}\left(d_{i}\right) \rightarrow \operatorname{coker}\left(d_{i}\right)
$$

is isomorphic to the original two-term complex. $W_{i}$ is naturally extended (denoted by the same notation) to

$$
\bigoplus_{i} E_{i}^{0} \rightarrow\left(E_{i}^{1}\right)^{*}
$$

Choosing a Hermitian metric on $E_{i}^{1}$ defines an isomorphism $\bar{E}_{i}^{1 *} \cong E_{i}^{1}$. Define the Witten map to be the following:

$$
\mathbb{W}=\bigoplus\left(d_{i}+\bar{W}_{i}\right): \bigoplus_{i} E_{i}^{0} \rightarrow \bigoplus_{i} \bar{E}_{i}^{1 *} \cong \bigoplus_{i} E_{i}^{1}
$$

Let $\pi_{j}: \bigoplus_{i} E_{i}^{j} \rightarrow \mathcal{W}_{g, k, G}$ be the projection map. The Witten map defines a proper section (denoted by the same notation) of the bundle $\mathbb{W}: \bigoplus_{i} E_{i}^{0} \rightarrow$ $\pi_{0}^{*}\left(\bigoplus_{i} E_{i}^{1}\right)$. The above data defines a topological Euler class $e\left(\mathbb{W}: \bigoplus_{i} E_{i}^{0} \rightarrow\right.$ $\left.\pi_{0}^{*}\left(\bigoplus_{i} E_{i}^{1}\right)\right)$. Then,

Theorem 5.2.1 (Topological Euler Class Axiom).

$$
\begin{aligned}
& {\left[\mathcal{W}_{g, k, G}\left(\gamma_{1}, \ldots, \gamma_{k}\right)\right]^{v i r}=(-1)^{\chi} e\left(\mathbb{W}: \bigoplus_{i} E_{i}^{0} \rightarrow \pi_{0}^{*}\left(\bigoplus_{i} E_{i}^{0}\right)\right)} \\
& \quad \cap\left[\mathcal{W}_{g, k, G}\left(\gamma_{1}, \ldots, \gamma_{k}\right)\right],
\end{aligned}
$$

where $\chi=\sum_{i}\left(\operatorname{dim}\left(E_{0}\right)-\operatorname{dim}\left(E_{1}\right)\right)$.

The above axiom implies two subcases.

(1) Concavity: Suppose that all marked points are Neveu-Schwarz and are decorated with $1 \in H^{N_{\gamma_{i}}}\left(\mathbb{C}_{\gamma_{i}}^{N}, W_{\gamma_{i}}^{\infty}, \mathbb{Q}\right)=\mathbb{C}$. If $\pi_{*}\left(\bigoplus_{i=1}^{N} \mathbb{L}_{i}\right)=$ 0 , then the virtual cycle is given by capping the top Chern class of the orbifold vector bundle $R^{1} \pi_{*}\left(\bigoplus_{i=1}^{N} \mathbb{L}_{i}\right)$ with the usual fundamental cycle of the moduli space:

$$
\left[\mathcal{W}_{g, k, G}\left(\gamma_{1}, \ldots, \gamma_{k}\right)\right]^{v i r}=c_{\text {top }}\left(-R^{1} \pi_{*} \bigoplus_{i=1}^{N} \mathcal{L}_{i}\right) \cap\left[\mathcal{W}_{g, k, G}\left(\gamma_{1}, \ldots, \gamma_{k}\right)\right]
$$

(2) Index zero: Suppose that $\operatorname{dim} \mathcal{W}_{g, k, G}\left(\gamma_{1}, \ldots, \gamma_{k}\right)=0$ and all the marked points are Neveu-Schwarz. If the pushforward $\pi_{*}\left(\bigoplus \mathbb{L}_{i}\right)$ and $R^{1} \pi_{*}\left(\bigoplus \mathbb{L}_{i}\right)$ are both vector spaces of the same rank, then the virtual cycle is just the degree $\operatorname{deg}(\mathbb{W})$ of the Witten map. 
It turns out that all the required 4-point correlators belong to concave cases where the axiom applies. To compute the top Chern class of the obstruction bundle, we need to use the orbifold Grothendeck-RiemannRoch formula. Conceptually, it is straightforward. The actual computation requires keeping careful track of fractions! All of the cases have been worked out in $[$ FJR2] (see another treatment $[\mathbf{C}]$ ). For 3-point correlators, not all the cases are Neveu-Schwarz. But in the ADE-case, the 3-point correlators from the Neveu-Schwarz sectors completely determine the 3-point correlators with Ramond insertion by associativity. Apparently, the phenomenon that Neveu-Schwarz sectors determine everything occurs also beyond ADEsingularities.

When we match the 3-point and 4-point correlators of the A-model and the B-model, another interesting phenomenon arises. Recall that the primitive form (say for the DE-series) is $c d x d y$ where $c$ is an arbitrary constant. Apparently, the A-model theory singles out a particular constant $c$ on the B-side. For general singularity theory, there should be a moduli of primitive forms. In mirror symmetry (see more in the next section), the A-model theory should correspond to a particular point of the moduli of primitive forms on the B-side.

Finally, we comment on the proof of the topological Euler class axiom. The topological Euler class axiom is quite obvious if we set up our theory using the smooth metric at marked points and nodal points. Unfortunately, it is quite difficult to handle the Ramond sector in the smooth theory. It took the authors several years to realize that the right choice of metric for the Ramond sector is the cylindrical metric (cylindrical theory). This is where the general theory was set up and its formal Gromov-Witten theory properties were proven. The proof of the topological Euler class axiom is basically a proof of equivalence of the smooth theory and the cylindrical theory in the case of Neveu-Schwarz sectors. It takes considerable work and is probably the most difficult part of the entire construction. Nevertheless, the topological Euler class axiom reduces the calculation to algebraic geometry where many more techniques can be applied. It remains a difficult problem to give a completely algebraic treatment for the Ramond sector.

5.3. ADE-singularities and Mirror symmetry. Now, we focus on 3 -point correlators. It is well-known that 3-point correlators define a quantum ring structure on $\mathcal{H}_{W, G}$. To calculate 3 -point correlators means to calculate the quantum ring structure. We single out the 3-point case for two reasons. First, this is the starting point of the proof of Witten's ADE-hierarchies conjecture. Secondly, this is the subject of Witten's other conjecture.

ADE-Self-Mirror Conjecture: ADE-singularities are self-mirror in the sense that $\mathcal{H}_{W,\langle J\rangle}$ are ring isomorphic to $\mathcal{O}_{W}$ for $A D E$-singularities $W$.

It was a surprise to the authors initially when the ADE-self mirror conjecture turned out to be the first step towards the ADE-integrable hierarchies conjecture. It is even more surprising that this conjecture fails for $D_{n}, n$ odd. 
The search for the mirror of $D_{n}$ leads to the singularity $D_{n}^{T}=x^{n-1} y+y^{2}$ and a much better understanding of mirror symmetry of singularities in general. More precisely, the statement of the theorem is

TheOrem 5.3.1. (1) Except for $D_{n}$ with $n$ odd, the ring $\mathcal{H}_{W,\langle J\rangle}$ of any simple (ADE) singularity $W$ with symmetry group $\langle J\rangle$ is isomorphic, as a Frobenius algebra, to the Milnor ring $\mathcal{O}_{W}$ of the same singularity. For all of these rings the element $J$ generates the entire maximal diagonal symmetry group $G_{\max }$.

(2) The ring $\mathcal{H}_{D_{n}, G_{\max }}$ of $D_{n}$ with the maximal diagonal symmetry group $G_{\max }$ is isomorphic, as a Frobenius algebra, to the Milnor ring $\mathcal{O}_{x^{n-1} y+y^{2}} \cong \mathcal{O}_{A_{2 n-1}}$.

(3) The ring $\mathcal{H}_{D_{n}^{T}, G_{\max }}$ is isomorphic, as a Frobenius algebra, to the Milnor ring $\mathcal{O}_{D_{n}}$ of $D_{n}$.

The proof of the above theorem is carried out via the index zero case of the topological Euler class axioms and associativity.

We want to mention that Kaufmann [Ka1] also proved a version of Witten's ADE self-mirror conjecture using an entirely different definition of A-model ring.

\section{Beyond ADE}

As we mentioned in the introduction, ADE-singularities comprise a special class of singularities. On the Kac-Moody algebra side, there are $B_{n}^{(1)}, C_{n}^{(1)}$, $F_{4}^{(1)}$-series. They should correspond to simple boundary singularities. For other more exotic creatures such as $G_{2}^{(1)}, A_{n}^{(2)}, E_{6}^{(2)}, D_{4}^{(3)}$, I do not know if they correspond to singularity theory. Another interesting class of examples are the integrable hierarchies associated to elliptic curves [DJKM5]. Recall that we have three classes of elliptic singularities

$$
P_{8}=x^{3}+y^{3}+z^{3}, X_{9}=x^{4}+y^{4}, J_{10}=x^{3}+y^{6} .
$$

Do these three classes of elliptic singularities correspond to elliptic integrable hierarchies in [DJKM5]?

Another very interesting class of singularities are Arnold's exceptional singularities

$$
\begin{aligned}
& : E_{12}=x^{3}+y^{7}, E_{14}=x^{3}+y^{8}, Z_{12}=x^{3} y+x y^{4} . \\
& : W_{12}=x^{4}+y^{5}, Q_{10}=x^{3}+y^{4}+y z^{2}, Q_{12}=x^{3}+y^{5}+y z^{2} . \\
& : S_{12}=x^{2} y+y^{2} z+x z^{3}, E_{13}=x^{3}+x y^{5}, Z_{11}=x^{3} y+y^{5} . \\
& : Z_{13}=x^{3} y+y^{6}, W_{13}=x^{4}+x y^{4}, Q_{11}=x^{3}+y^{2} z+z x^{3} . \\
& : S_{11}=x^{4}+y^{2} z+x z^{2}, U_{12}=x^{3}+y^{3}+z^{4} .
\end{aligned}
$$

These singularities have a deep connection to hyperbolic geometry in the same way as ADE-singularities have a deep connection to Euclidean geometry. It is not inconceivable that the exceptional singularities have some 
integrable hierarchies behind them. This raises an interesting question for experts in representation theory and integrable hierarchies.

It is clear that the subjects of singularity theory and integrable hierarchies are wide open. Our experience in the ADE case tells us that the first step towards integrable hierarchies is to understand the mirror symmetry of singularities. Here, a great deal of progress has been made due to the work of Krawitz and his collaborators $[\mathbf{K r}]$ for the invertible singularities.

Almost twenty years ago, the physicists Berglund-Hübsch proposed the following elegant mirror symmetry construction for an invertible singularity. Let $M_{W}$ be the matrix of exponents. Namely, if $W=\sum_{j=1}^{N} c_{j} \prod_{i=1}^{N} z_{i}^{b_{i j}}$, $M_{W}=\left(b_{i j}\right)$. The invertibility implies that $M$ is a square matrix. BerglundHübsch proposed that the mirror singularity $W^{T}$ is another invertible singularity with the property $M_{W^{T}}=M_{W}^{T}$. To complete the story, for any $G \subset G_{\max }(W)$, we should also construct a dual group $G^{T} \subset G_{\max }\left(W^{T}\right)$. This was not entirely clear in the literature except in some special cases. Recently, Krawitz has constructed such a dual group $G^{T}$. His construction is a little bit involved and we will refer readers to his paper. Here are some important special cases.

$$
G_{\max }^{T}=\{1\},\langle J\rangle^{T}=S L \cap G_{\max }\left(W^{T}\right) .
$$

Here $S L \cap G_{\max }\left(W^{T}\right)$ is the subgroup of $G_{\max }\left(W^{T}\right)$ with determinant one. Furthermore, he showed that $G^{T}$ for any $G \subset S L \cap G_{\max }$ is admissible.

Then, Krawitz proved the following elegant theorem:

TheOREM 6.0.2. Suppose that $W$ is an invertible singularity and $G$ is an admissible group. Furthermore, we assume that $q_{i}<\frac{1}{2}$ for all $i$. Then $\mathcal{H}_{W, G}$ is ring isomorphic to $\mathcal{O}_{W^{T}, G^{T}}$.

The special cases of singularities with modality two were computed previously by Krawitz and his collaborators. When $W$ is an Arnold exceptional singularity and $G=\langle J\rangle$, it recovers Arnold's strange duality.

A particular useful case for integrable hierarchies is the mirror symmetry

$$
\mathcal{H}_{W, G_{\max }} \cong \mathcal{O}_{W^{T}}
$$

Notice that $\mathcal{O}_{W^{T}}$ is the part of larger theory in terms of Frobenius manifold and Givental formal Gromov-Witten theory $\mathcal{D}_{W^{T} \text {, formal }}$. It is natural to conjecture

Conjecture I: For an invertible singularity $W$,

$$
\mathcal{D}_{W}=\mathcal{D}_{W^{T}, \text { formal }}
$$

up to a linear change of coordinates for a particular choice of primitive form of $W^{T}$.

Conjecture II: For an invertible singularity $W, \mathcal{D}_{W}$ is a $\tau$-function of the $W^{T}$-hierarchy. Here, the $W^{T}$-hierarchy is the hierarchy (remains to be constructed for the general case) corresponding to the Dynkin diagram of $W^{T}$. 


\section{References}

[ADKMV] M. Aganagic, R. Dijkgraaf, A. Klemm, M. Mariño, and C. Vafa, Topological strings and integrable hierarchies, Comm. Math. Phys. 261 (2006), no. 2, 451-516.

[AGV] V. Arnold, A. Gusein-Zade, and A. Varchenko, Singularities of differential maps, vol I, II, Monographs in Mathematics, Birkhauser, Boston, 1985.

[BH] P. Berglund and T. Hübsch, A Generalized Construction of Mirror Manifolds, Nucl. Phys. B 393 (1993) 377-391.

[C] A. Chiodo, Towards an enumerative geometry of the moduli space of twisted curves and rth roots. To appear in Compositio Mathematicae. Preprint: math.AG/0607324.

[CR1] A. Chiodo and Y. Ruan, LG/CY correspondence of quintic 3-fold via symplectic transformation, arXiv:0812.4660.

[DJKM1] E. Date, M. Jimbo, M. Kashiwara, and T. Miwa, Operator approach to the Kadomtsev-Petviashvili equation, Transformation groups for soliton equations, III, J. Phys. Soc. Japan 50 (1981), 3806-3812.

[DJKM2] E. Date, M. Jimbo, M. Kashiwara, and T. Miwa, A new hierarchy of soliton equations of KP-type. Transformation groups for soliton equations. IV, Phys. D4 (1982), 343-365.

[DJKM3] E. Date, M. Jimbo, M. Kashiwara, and T. Miwa, Transformation groups for soliton equations. Euclidean Lie algebras and reduction of the KP hierarchy, Pub. Res. Inst. Math. Sci. 18 (1982), 1077-1110.

[DJKM4] E. Date, M. Jimbo, M. Kashiwara, and T. Miwa, Transformation groups for soliton equations, Proc. Res. Inst. Math. Sci. Symposium, M. Jimbo and T. Miwa, eds, World Scientific, 1983.

[DJKM5] E. Date, M. Jimbo, M. Kashiwara, and T. Miwa, Landau-Lefschitz equation: solitons, quasi-periodic solutions and infinite-dimensional Lie algebras, J. Phys. A: Math. Gen. 16(1983) 221-236.

[Di1] R. Dijkgraaf, Topological field theory and $2 D$ quantum gravity. Twodimensional quantum gravity and random surfaces (Jerusalem, 1990/1991), Jerusalem Winter School Theoret. Phys., 8, World Sci. Publ., River Edge, NJ, 1992, pp. 191-238.

[Di2] R. Dijkgraaf, Intersection theory, integrable hierarchies and topological field theory. New symmetry principles in quantum field theory (Cargse, 1991), NATO Adv. Sci. Inst. Ser. B Phys., 295, Plenum, New York, 1992, pp. 95-158.

[DS] V. Drinfeld and V. Sokolov, Lie algebra and equations of KDV type, Itogi Nauki Techniki 24 (1984), 81-180.

[FSZ] C. Faber, S. Shadrin, and D. Zvonkine, Tautological relations and the r-spin Witten conjecture, arXiv:math/0612510.

[FJR1] H. Fan, T. Jarvis, and Y. Ruan, Geometry and analysis of spin equations, arXiv:math/0409434.

[FJR2] H. Fan, T. Jarvis, and Y. Ruan, The Witten Equation and Its Virtual Fundamental Cycle, arXiv:math/0712.4025.

[FJR3] H. Fan, T. Jarvis, and Y. Ruan, The Witten equation, mirror symmetry and quantum singularity theory, arXiv:0712.4021.

[FGM] E. Frenkel, A. Givental, and T. Milanov, in preparation.

[Gi1] A. Givental, Symplectic geometry of Frobenius structures, Frobenius manifolds, Aspects Math., E36, Vieweg, Wiesbaden, 2004, pp. 91-112.

[Gi4] A. Givental, Gromov-Witten invariants and quantization of quadratic Hamiltonians, Dedicated to the memory of I. G. Petrovskii on the occasion of his 100th anniversary, Mosc. J. 1 (2001), no. 4, 551-568, 645. 
[GM] A. Givental and T. Milanov, Simple singularities and integrable hierarchies, The breadth of symplectic and Poisson geometry, Progr. Math., 232, Birkhauser Boston, Boston, MA, (2005) 173-201.

[HM] T. Hollowood and J. Miramontes, Tau-functions and generalized integrable hierarchies, Comm. Math. Phys. 157, 99-117 (1993).

[IV] K. Intriligator and C. Vafa, Landau-Ginzburg orbifolds, Nuclear Phys. B 339 (1990), no. 1, 95-120.

[J] P. Johnson, Gerbe and equivariant $G W$-theory of orbifold $P^{1}$, preprint.

[Ka1] R. Kaufmann, Singularities with symmetries, orbifold Frobenius algebras and mirror symmetry, Contemp. Math., 403 (67-116).

[Ka3] R. Kaufmann, Orbifolding Frobenius algebras, Internat. J. Math. 14 (2003), no. $6,573-617$.

[KW] V. Kac and M. Wakimoto, Exceptional hierarchies of soliton equations, Proc. Sym. in Pure Math. Vol 49(1989), Part 1, 191-237.

[AK] A. Klemm, S. Theisen, and M. Schmidt, Correlation functions for topological Landau-Ginzburg models with $c=$ 3. Int. J. Mod. Phys. A7:6215-6244, 1992.

[Ko] M. Kontsevich, Intersection theory on the moduli space of curves and the matrix Airy function, Comm. Math. Phys. 164 (1992), 1-23.

[KM] M. Kontsevich and Y. Manin, Gromov-Witten classes, quantum cohomology, and enumerative geometry, Commun.Math.Phys. 164 (1994) 525-562.

[Kr] M. Krawitz, FJRW-ring and mirror symmetry of singularities, preprint.

[KR] M. Kreuzer, On the classification of quasihomogeneous functions, Comm. Math. Phy. 150 (1992), 137-147.

[MT] T. Milanov and H-H. Tseng, Equivariant orbifold structures on the projective line and integrable hierarchies, arXiv:0707.3172.

[NY] M. Noumi and Y. Yamada, Notes on the flat structures associated with simple and simply elliptic singularities, in "Integrable Systems and Algebraic Geometry," eds. M.-H.Saito, Y.Shimizu, and K. Ueno, World Scientific, (1998), 372-383.

[PV] A. Polishchuk and A. Vaintrob, Algebraic construction of Witten's top Chern class, Advances in algebraic geometry motivated by physics (Lowell, MA, 2000), Contemp. Math., 276, Amer. Math. Soc., Providence, RI, 2001, pp. 229-249.

[PR] P. Rossi, Gromov-Witten theory of orbicurves, the space of tri-polynomials and symplectic Field Theory of Seifert fibrations, arXiv:0808.2626.

[S] K. Saito, Primitive forms for a universal unfolding of a function with an isolated critical point, J. Fac. Sci. Univ. Tokyo Sect. IA 28 (1982).

[Te] C. Teleman, The structure of 2D semi-simple field theories, preprint 2007, arXiv: 0712.0160 .

[Wa1] C. T. C. Wall, A note on symmetry of singularities, Bull. London Math. Soc. 12 (1980), no. 3, 169-175.

[Wa2] C.T.C. Wall, A second note on symmetry of singularities, Bull. London Math. Soc. 12 (1980), no. 5, 347-354.

[Wi1] E. Witten, Two-dimensional gravity and intersection theory on the moduli space, Surveys in Diff. Geom. 1 (1991), 243-310.

[Wi2] E. Witten, Algebraic geometry associated with matrix models of twodimensional gravity, Topological models in modern mathematics (Stony Brook, NY, 1991), Publish or Perish, Houston, TX, 1993, 235-269.

[Wi3] E. Witten, private communication.

[Wi4] E. Witten, Phases of $N=2$ Theories In Two Dimensions, Nucl. Phys. B 403 (1993), 159-222. 
